\title{
CHIT: A Cost Accounting Program for Postirradiation Examinations of Fast Breeder Reactor Materials
}

by

J. R. Phillips

K. E. Dowler 
Printed in the United Stoten of America. Available from Notional Technical Information Service

US Departmont of Commerce

5285 Port Royal Road

Springtiold, VA 2215]

Pricn: Printed Copy S4.00 Microliche $\$ 2.25$

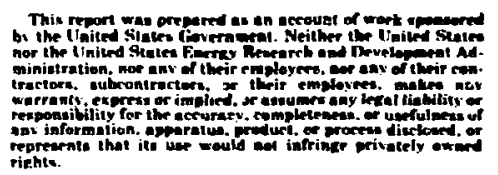

reprews. 


\section{CHIT: A COST ACCOUNTING PROGRAM FOR HOSTRRADLATION EXAMINATIOSS OF} FAST GREEDER NEACTOR MLATERLALS

by

\author{
J. R. Phlllips and $k$. E. Dowler
}

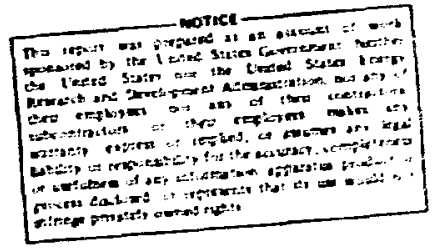

\begin{abstract}
CHIT is the Los Alamos Sctentifie Laboratory's cost accounting computer program for nondestructive and destruetlie examinations of Irradiated fuel pins. The program aliows immedinte retrieval of fuel pin examination informatlon and provides ttemized listings for completed and projected fuel pla examInations, detailed cost accounilng summaries for each investigator, fuel pin eximinatlons buring a speeified time interval, and varlous subsets of the information. CirT has been in sucessful oxeration for the past two years, providling precise information on cost accounting more efficiently than possible with a manual technique.
\end{abstract}

\section{TNTRODUCTION}

The Chemistry-Naterials Solence Division and the Nondestructive Testing Group of the Iynamic Testing Ditision of the Los Alimos Selentific Laborntory (LASL), perfor $m$ postirradiation examinations of experimental fast reactor fuel pins for the Division of Reactor Research and Development (RKL) of the Energy Research and Developm(ant Administiation (fHIDA). RRI) divides fiscal yenr furding for this wort anong several principal experimentc1:; depending on thetr projected examination requirenitonts for that fiscal yerc. The amount allocated to ench cxperimenter is ealled his "chit" (a term suggested by an IRTD empioyee), from which derives the name of the cost accounting computer program.

The need for an accounting program became appa:ent with the increase in number of experimenters, examinations po:formed, and LASI, groups performing the examinatlons. To standardize the levels of effort involved in postirradiation examinations of pins by varlous coi.'ractors, IRD suggested five separate classes. These class- es, defined by types of examinatlons routinely performed at LASL, are detatley in Appendix A. F:xaminations that do not fall within any particular class are considered special examinatlons; cxaminations that were not included in the experimenter's originat examination plan are called additional examfnations. Relative costs have been assigned to each eximination class as shown in Appendix B. These costs are useful for budiget planning and for CIIT determination of cost orerruns for any particular pin examination. Note that all costs in this report hase been used for tllustrative purposes only and are not actual cests.

\section{PirocinaN Deschipton}

The CIIT cost accounting computer program calculates projected and accrued costs of all experimental fuel pin exam Inat Jons at LASL. The accrued costs are obtained from four sroups: Annlytlcal Chemistry Group. Plutonium Chenlstry and Metallurgy Group, Irradiated Interials Examination and liardling Group, and Nonde- 


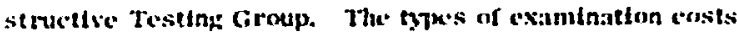
collectively pertormed ba these froups are Ilsterl in ipjenatx C. Fach examinatlen has bech assigned a worl: unlt value that can be rolated to actual dallars by using a untegue Internal converston factor for each grmify.

The sumr.e-ton of these eximinatlon cosis is corrected automatically for the variable support activities, resulting in actual costs per eximination and total cost for the complete examination of each pin. The sujport activilics a re sartable (nuctuating slightly from month to month) because of scheduled preventive mainterance and unschectuled repairs. Thersfore, at the end of the fiscal year, each cost center fexperimenteri will have becn chirged the correct fraction of the nonexamination costs.

Specific informition shout indivitual fuel pins is stored on an L'P! tion of the data. IPUATE is a computer program for manjpulating data files and is avaibale on most computing systems. The program runs un a Control Dala Corporaton 7600 compucer. requi res less than $1-$ min cent ral processing unit time,and costs less than $\$ 10$ for a program in which all options are rexuested.

cilit offers six maln options.

- Full tisting. A complete listing of ench fucl pln inclueling types of exinination. dates of raquest for examinntion, completion dates, actual cests, preitous fisea] ycar's costs ncerucd, and present nseal year totals. - Special Fienmations. An itenized listing of the special examinations performed turing the specifled time interval that do not fall into the fite classes clesctrbed in Appentix A.

- Additional Examinations. A listing of the cxaminations performed during the stated pertal that were not a pirt of the experimenter's orfginal test plan.

- List Data. The data on the t:PDATE fitc is copled to the output. nroviding a detalled image of all the Informatjon stored on the data fle.

- Summary. Summaries of the accrued costs of all the experimenters for the flscal year to date, or any specIffed time Interval, including scparation of spectal and addittonal cxaminations and a cetailed sumianry listing for nll the fuel pins and cost overruns in excess of those costs

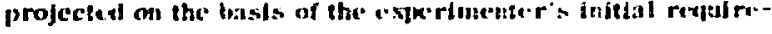
ments.

- Liximinatlon Group. Itemiged Itsting of the examinattous perfertied ly the four organlzational sroups thering the specifted dates.

These options may be exectutcof on an artual wear(o-date) or quarterly basis to obtaten summarites of the experimenter's actinilas and Indisfetual fucl pln rata for reporting forogress to the cexperimenters and to fills. The date file is motified at leafi monthly: if an cxperimenter has specific questions about the progress of a partleular fucl pin. the fiformation is readlly avathable. This Iuformallon can bx used by expertinenters for adjusting thelr cxamination requests to correspond to thelr remaining chit.

CIIfl asfumes that all monles or total chits will be

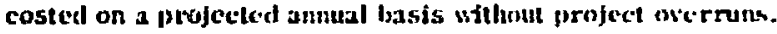
The total chit is divfded by the number of ctys la the year

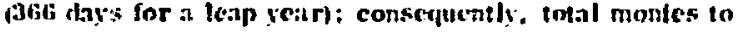
he costud thrting any reporting porlod will ix the fraction of the total chit that the particulis reporting gertort represents. A firgt pass is made through the dita to fetermint: a nututiping ftetor. called the support factor. which is then used to adjust all costs to bring the total for the reporting perted exual to the pr per fraction of the sotal chit. This support fetor represents costs for the stoportInf; groups and nctivitles, such as quallty thosurance, cealpment malitenance and repatr. and other fnctirect habor custs. The support factor reflects the performance and effelency of the entire operation, c. R. , beklog oi pins. frompt delivery of required forms and inf.cmation by experinenters. dountime renulred for routine maintenance and cmergency repalrk to cximination and support equlpwent, and proper sehechuling of pins through examination sequence.

A complete listing of the control cards, a listing of the prograin's main wrables, and a FolrTRAx IV listing of the computer code are presented in Appendixes $\mathrm{V}$. E, and F. respectively. Comment cards have been liberally Inserted in the source deck to explain the options and vartables of the cost accounting program.

Typical examples of program output are presented in 
Apux'ndixes $(;-1 .$. Apuendix (i gives a summary of accrucel costs of all the expeztmenters for the fiscal year to aly specified riporting date. Costs glver in the Appendixes are hypothetical; the total of the experimenters chits was arbitravily set at sil million. An expetimenter's chit is fixed for the fiseal year based on hi, approved profected aximination repuirements. The commelted costs a) determinced lay a nore detalled eximination plan submitted huring the fiscal year. Committed and costed values are pereentughes of committed costs and actual costs it relation to the expertmenter's chit. The summary also sepalates projected aml actual costs for addtional extminations.

A simblar summaty of experimenter cost aceiual is presented In Apuentis It which breaks down exyerimenter's costs un a guartorly period lasis. Commited costs have Iren unilled in this summary because fiscal year commitmests do not conform well to a quarterly ferlod. Both summarics are viltable in reflecting the status of each cxperineuter"s chit chring the spectfed reporting period.

Tutal costs and status of ench pin are summartzed In the outjul presented in Appendix 1 . Costs of examfuations performed chring the present fiseal year as well as total cost s for all previous ycars are presented. In additon, a cliss cost overiun is presented whtch represents the difference between the actual exmmination costs and the estimited costs for a particular examination class when the actual costs are greater tian the class costs shown in Appendix 13 . The overizias are usually the results of an experinenter requesting additional exmminations that are not defined in a paticular class. Sote that sume fuel pins have not been asiglgned to a class, ustally the result of an incomplete examination plan. Often an experimenter wishes to evajuate preliminary results before committing himself to a complete plan.

Apyendlx $J$ presents a summary of only those pins for which all planned examinations have been completed during a quarterly period. This trre of summary can he produced to fnclude all pins that are completed during the tiscal year.

An example of a summary for special ex.minations completed during a quarterly period is presented in
Aprendix $K$. The same type of summan can also it printed for additional examinations; both the special and additional cxmination summaries can be listed for yearto-date and fiscal year periods.

The option for a full llsting gtves a complete data listing for rach pin including type of cxamination. date of request for exilifination, exam!nation completion date. unit costs, total costs, presious flscal year costs, and present fiscal yes: totals. This listing, shown in Apper:dix $I$. is valuable for determsining in detall the stat is of any particular fual pín.

\section{1?. CONC1.LSIONS}

CiIr, tlie cost accounting computer program for texamining experfmental fucl pins, has been in operation at LASL for 2 yr. Clilt provid s project administrators and experimenters with summaries a: d detafied listings of profected and accrued costs of specific exminations. Before CiflT was available, cost projections for specific or gencxal cximinations were based primarly on experfence and Intuition. Now Indiridual costs have been accurately quar tified, permitting IASL to administer the project more efficientiy and allowing experimenters to make realistl. profections based on more accurate est3mates. Operation of the entire cost a:counting prograin requizes aibout :man-alays per month, whereas a comparable manual technique for a project of this size would require nany times the manpower effort. This general type of cost accounting can be readily adapted for use at other fuel pin examination fachities.

\section{ACKNOWLEUGEMENTS}

The CHIT cost accounting computer program has benefited from the suggestions of $R$. D. Baier, ChemistrMaterials Science Division leader, and the following principal investlgators of the project: J. W. Schulte.

G. R. Waterbury, and K. A. Johnson. W. T. Wood assteted in collecting necessary Information and offered manyhelpful suggentions. CHTT was developed at the request of the Division of Reictor Research and Development of the Energy Besearch and Development Administration. 


\section{APPE:DES A \\ POSTIRRALIATION EXAMTNATIONS AND SUPPORT OPERATIONS \\ PERFCRMED AT THE LAST HOT CELL FACILITIES}

1. Cask unloading

2. Visual Inspection. QC inspection: Identification, radiation, contamination, temperature.

3. Betatron radiography. Capsule or pin at $0^{\circ}$ and $90^{\circ}$; furnish negatives and prints.

4. Gross gamma scan. Capsule or pia. All energies $>150 \mathrm{keV}$, one axial and three diar.etral scans.

5. Photography (capsule)

Full-length $\theta^{\circ}$ and at maximum bow.

Inc remental, three orientations at $2 x$, if required.

Cinusual areas.

6. Profilometry (optical)

Capsiale. Up to five or ientations, if requested.

Pin. Up to five orientations, if requested.

7. Gas sampling and mass spectrometric analys is of capsule cover gas; quantity of gas and void volume calculations.

8. Capsule disassembly. Remove sodium bond, wire wrap, capsule clad. Dispose of hardware. (Breached pins would be ot sassembled in an alpha containment bc.: where the manpower requirements ire doubled.)

9. Profllometry on breashed uin (mechanical). Orientations as requested.

10. Photography (pin)

Full-length, $0^{\circ}$ and maximum iow.

Incremental, three orientations at $2 \mathrm{X}$, If requested.

Unusual areas.

\section{CLASS II}

\section{All of Class I}

2. Fission gas (pia). Sa:apling, analysis, volume, and quantity calculations.

3. Sectioning

4. Sodium dietillation

5. Microstructural analysis. Two fuel-clad samples, each with

a. Macro-photographs. b. Autoradiographs (alpha and beta-gamma).

c. Ten selected microscopy photographs, one print each of items 1 and 2.

d. Mosaic.

e. One high-magnification strip.

6. Burnup (1 per pin)

\section{CLASS III}

1. All of Class I

2. All of Class $\Pi$ except item 5

3. Microstractural analysis

Three Kel-clad specimens.

Same -xsimination as Class $\mathrm{I}$, item 5. a-e.

4. Multispectral gamma scan (four isotopes, 5-mm steps). One axial scan.

5. EMX (one sample). Characterize fuel-clad interface at two points; radial $U / P u$ ratlo, two scans.

b. Density (two samples)

\section{CLASS IV}

1. All of Classes L, IL, and III (except ftem 3)

2. Microstructural analysis

Four fuel-clad specimens.

Same as Class $\Pi$, item 5. a-e.

3. Multispectral gamma scan (ten ifotopes, $0.5-\mathrm{mm}$ steps)

4. ENDX (one sample). Characterize fuel-clad interface at three polnts; radial U/Pu ratio; radlal di: tribution of four fission products, two scans. 
1. Includes all of Cluss $T V$ and ustually several parts of items 2 or 3 below.

2. Special exanilnations (examples)

a. Capsule or pin weight

b. Balance point

c. Clad or fuel SEM (one sample)

d. Length measurement

e. Location of pin fallure point

f. Oxygen in clad

g. Oxygen in fuel

h. Clad microhardness (one sample)

1. Fuel-clad replication (one sample)
3. Additional examinatton requests (examples)
a. Buraup sample
b. Retained fission gas
c. TWODM gamina scan four isotopes, one loca- tion)
d. Profilometry tone pia, multiscan, data redic- tion)
e. Microstruetural analysis, (one sample)
f. Burnup profile from gamma scan
g. Clad leach, cesium analysis
h. EMX (extensive examination of one or more samples)

APPENDEX B

COST OF POSTIRRADIATION EXAMINATIONS

\begin{tabular}{crr} 
Class & \multicolumn{2}{c}{ Cost in Thousands of Dolla } \\
Examination & Unbreached Pin & Breached \\
\cline { 2 - 3 } I & 3.1 & 4.6 \\
II & 4.5 & 10.1 \\
III & 11.6 & 13.6 \\
IV & 16.1 & 18.5 \\
V & 32.0 & 32.0
\end{tabular}

absolute dollar values listed are hypothetical, but relative costs are consistent, based on LASL cost accounting for the past $2 \mathrm{yr}$. 
APPFEDX 6

FeEL-PIX EXAMTATIONS

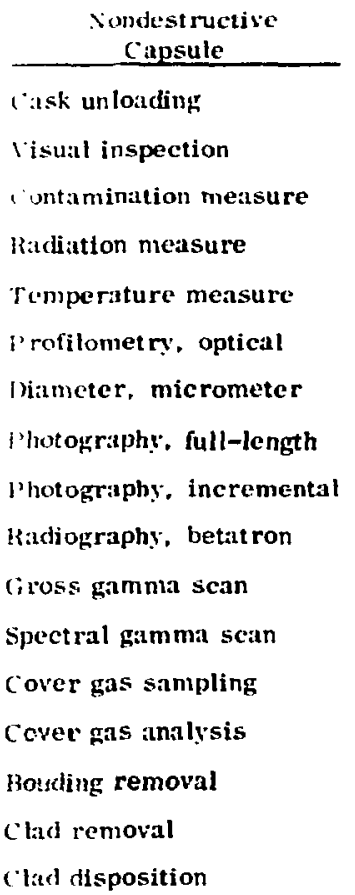

\begin{tabular}{l}
$\frac{1}{\text { Dest ructive }}$ \\
\hline Wire-urap removal \\
Wire-urap disposition \\
Fission gas sampling \\
fission gas analysis \\
Sectioning \\
Microstructural analysis \\
Microprobe preparation \\
Microprobe analysis \\
Burnup analysis \\
Clad density preparation \\
Clad density \\
Disposition of fuel \\
Disposition of pin clad
\end{tabular}

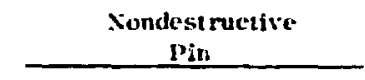

Visual inspection

Contamination measure

Hadiation measure

Temperature measure

Proftlometry, optical

Diameter measure

Radiography, betatron

Gross gamma sean

Spect ral gan:iais segi

Photography, full-length with wire

Photography, full-length without wire

l'hotography: incremental with wire

photography. incremental without wire

Fidy current scan

l'rofilomet rỵ: mechanical
Special Exarninations

Hetained fission gas

i ranium. plutonium determination

Flux tube storage

Weld leak check

I.cach gas plenum

Burst test samples

oxygen anaiyis of fuel

length measurement

Fddy current

Nitrogen in fuel

carbon in fuel

Kollmorgen photogr:tphs

Sodium distillation

Split pin clad

pressure leatí test

leak rate test

Sequentlal surface preparation

Clad faflure location

rWonIAI gamma scan

Burnup profile

oxyge.. in clad

Capsule weight

Balance point

SF.M sectloning 


\section{CONTIOL CARDS}

\begin{tabular}{|c|c|}
\hline iontrol ciard & Explanation \\
\hline START DATE: & $\begin{array}{l}\text { Sprecifies the starting ciate of the re- } \\
\text { porting period. }\end{array}$ \\
\hline IAST IJATE: & $\begin{array}{l}\text { Specifies the ending date of the report- } \\
\text { ing period. }\end{array}$ \\
\hline QSTART & $\begin{array}{l}\text { Specifies the starting date for a quart- } \\
\text { erly reporting period. }\end{array}$ \\
\hline (1ENI) & $\begin{array}{l}\text { Specifies the ending date for a quart- } \\
\text { erly reporting period. }\end{array}$ \\
\hline FISCAI. ISTX & Specifies the fiscal year. \\
\hline CHITS & $\begin{array}{l}\text { Specifies the number of chits or exper- } \\
\text { imenters and the total chit for the } \\
\text { fiscal year. }\end{array}$ \\
\hline STMMALY & $\begin{array}{l}\text { l'roduces a brief summary of eateh } \\
\text { fuel pin. }\end{array}$ \\
\hline H1.1. I.ISTING & $\begin{array}{l}\text { Prorluces a full listing for each fuel } \\
\text { pin. }\end{array}$ \\
\hline 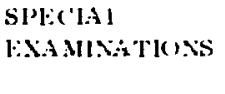 & $\begin{array}{l}\text { I'roduces a listing of spedial examin- } \\
\text { ations performed during the reporting } \\
\text { period. }\end{array}$ \\
\hline
\end{tabular}

\section{Control card \\ A DUITIONAI. EXAMTATKNS}

I.IST DATA

QLARTER

ANTIL

FXIPHIMTATER'S IDENTIFICATION

\section{APIENDLX E}

\section{VARLABIES}

Variable lame

\section{Alsta}

(AIII)

$\operatorname{xin} x$

Astrite

Xisplet:

UAAII)

QASIPEic

I.S'T

$A{ }^{\prime} \mathrm{T}$

NI:M

chIT l'in Irat:1

font rol cisul dital

l'rojected costs for additional examinations

Actual costs for additional examinations

I'rojected costs for special examinations

Artual costs for special examinations

Costs for additional examinations during quarterly period

costs for special examinations during quarter period

Totil estinuated costs for expirimenter

Actual costs for experimenter

Number of pins for experimenter

Experimenter's total chit
Variable vame

TCIT

PBCNT

[PIREST

PRTOT

CODE

QPRCNT

QACT

ICOPY

FYEAR

CMIBI-14 f. xplanation:

Produces a listing of a iditional examinations performed during the reporting period.

Produces a listing of in the input data $t s$ date. Used for uptatirag or changing data. This card overridee al! other listing or summary card commands.

l'roduces summaries and listing: performed or completed during the quarterly period.

Produces a summary of pins completed during the current fiscil year and a listing of all exismira tions completed by individua! groups during the fiscal iear.

Includes the experimenter's ritre. chit. and two-letter alpha-numeric code for program icentification.

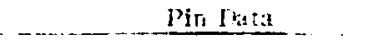 \\ Totz. costs for experimelicer}

Actual percentage of projecied costs for experimenter

Estimated percentage of projected costs for experimenter

Actual percentage of total dollars

Expe rimenter's identification cocie letters

Percentage of actual costs for experimenter during quarter

Actual costs for experimenter during quarter

Number of printed output copies required

Fiscal year

Group performing examination 


\section{APPENDX F}

\section{CIIT SOLRCE CONE (FORTRAN NN}

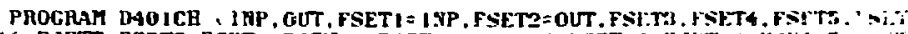

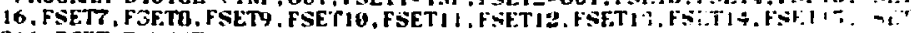

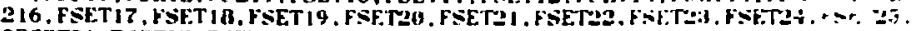

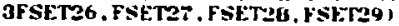

DIRENSION ADATAR:

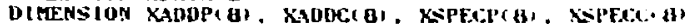

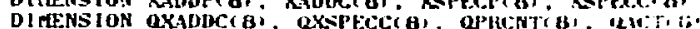

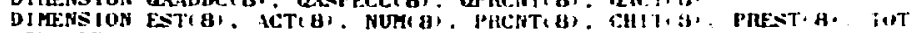
1(B). PITTTI B', SUP(B)

DI MENSION FACT( 10 )

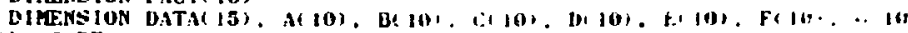
I). CODE ( 10

DI MENSION PY IOI. R (10)

DIMFNSION TITLE. Q. PHIN(S)

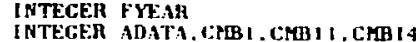

INTECER QSTAHT, UEND

INTECER A.B.C.D.F.F.G.DATES, DATE., F ISC,

INTEGER OS12,0534,0\$56, OE12. UE34.05J6

INTEGEK P. R

I NTEGER SUMLAHY

INTECER START, CHITS, FUIL, SPEC . WD

INTEGER CONE, EXPI

I NTEGER QUARTE

IN IEGER ANEUN.

I NTECER ANNCOMP

INTEGER PNACHB

INTTGER BLANK

INTESER CLANS, V1, U2, U3, U4, U5, B1, B2, B3, B4, B5

AEAL NDT

DATA gLANK1, 18-

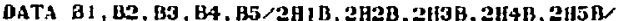

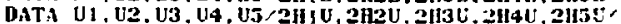

DATA' ISTBAT/ IOIN IST DATA,

DATA ASTEH3 - 4H***

DATA DEST 4 HDEST.

DATA NDT, ERP, PIN/ $I I N D T, 10 H-$ CAPSULE , ROH- PIN

DATA ASTER ASTER4/4H*F , 4fi***2/

DATA STAIT.LANT IOHSTART DATE. IOHLALT DATE，

DATA FISCAL 10HF ISCAL 197

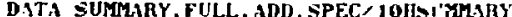

IA. IOHSPEC IAL F.X

DATA QUARTER THQUARTER

DATA ANNUAL GHANHUAL

UATA OSTART, OEND/GHOSTART, 4HOEND/

DATA CHB I IOUCYB-

DATA CMB $11 / 10$ C CMB- 1

DATA CRB 14/ IOHCRA- 14

DATA CHITS/10HCHITS

REWIND 2

K*\$स

PROGRAH IS SET UP TO MANIPULATE 8 SEPARATE FMYE:IIRETEHS

INPUT DATA REQUIRED AND OPTIONAL

START DATE 040174

LAST DATE O63074

OSTAR'T OZO174

CEND $\quad 193174$

FISCAL 1974

CulTs

SURMARY

FULL LIST INC

SPEC IAL EXAMINATIONS

ADDITIONAL EXAMINATIONS

QUARTER

ANNUAL

* HOTE* QUARTER ANB ANNUAL CONTROL CARDS GANHOT BE USED TOGE.EER SFM.

EXPER I YENTER

EXPERT FENTER

EXPER I MENTER

EXPER I MENTER

ACCOUMIING RUN FOR D401 0 Ú $/ 30 / 74$

FSET 3

FSET $\mathbf{5}$

FSET 6

FSET $?$

FSET $A$

FSET 9

FSET 10

FSET 11

FSET 12

FSET 13

FSET 14

1009000.

MEQUIRED

ZEOUIRED

RFOU I RED

FEOUIHED FOR QUARTER.Y

HEPORT

REOU I REU

RYOU I AED

OPTION AL

optional

OPTIONAL

OPT IONAI

OPTIUNAL

REQU I RED

238000 RFul': AFD

102000 RFUHIRED

300000 - AFOUIRFD

360006. RFouin

REQUIFF. SUTARY OF No. 3 EXPERIMENTS SUMMARY OF NO. \& EXPERIMENTS SUMMARY OF NO. 5 EXPERIMENTS SUMMARY OF NO. 6 EXPERIMENTS SUMRARY OF PS. 7 EXPERIMENTS SUMMARY OF NO. 8 EXPTRIMENTS LIST I NG OF NO. 1 EXPER I MFNTS LIST ING OF NO. 2 EXPERIMENTS LISTING OF NO. I EXPERIMENTS LISTING OF NO. 4 EXPEFIMENTS

LISTIYG OF NO. 5 EXPEAIMENTS 


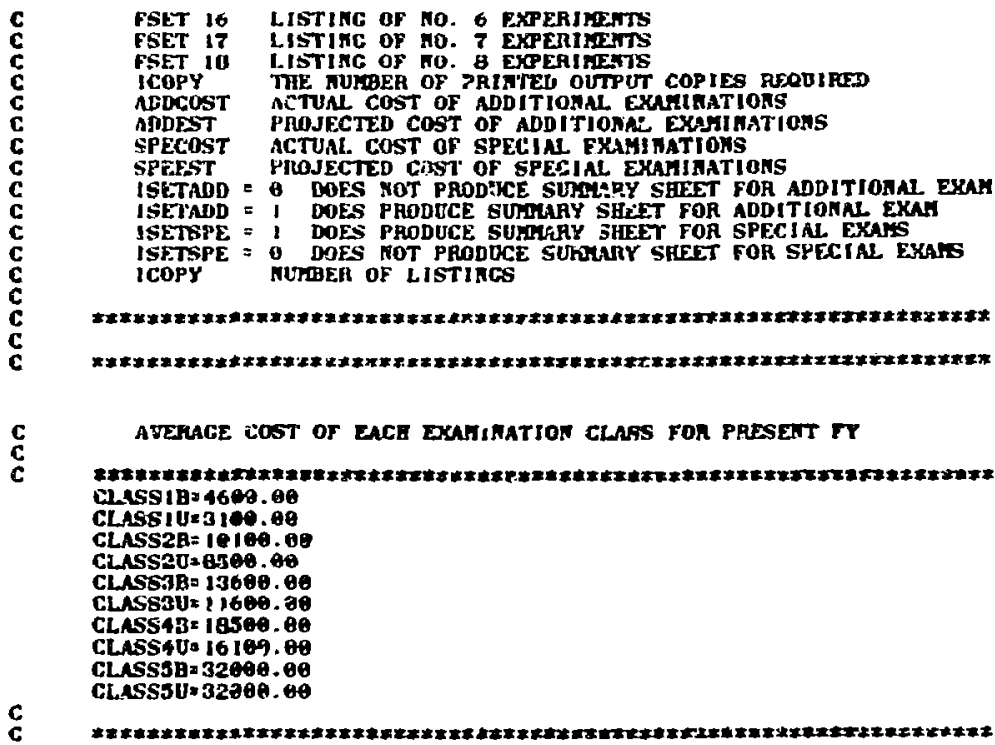

XAODCC II ACTUAL ADDITIOMAL COSTS FOR ITH EXPERIMEMTEA

XADDP( i) PROJECTED ADDITIONAL COSTS FOR 1TH EXPEAIROATER

XSPECP( I PROJECTED SPECIAL COSTS FOR ITH EXPERIMENTER

XSPECCC (I) PROJECTED ADDITIONAL COSTS FOR ITH EXPEAIRENTER

ESTI I TOTAL ESTIMATED COSTS OF ITH EXPERI YGATER

ACTI 1) ACTUAL ESTIMATS: COSTS OF ITH EXPEAIMENTE:

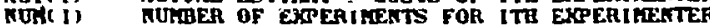

CBiti (1) DOLLAR VALUES OF 177 EXPEAIRENTERS CNIT

PRESTI I) VSTIMATED PERCENTAGE OF PROJEATED COSTS FOR ITE EXP

PRCAT I I ACTUZL OEACENTAGE OF PROJECTED COSTS FOR ITH EXF

PRTOT( I) ACTUAL PERCENTACE OF TOTAL DOLLARS

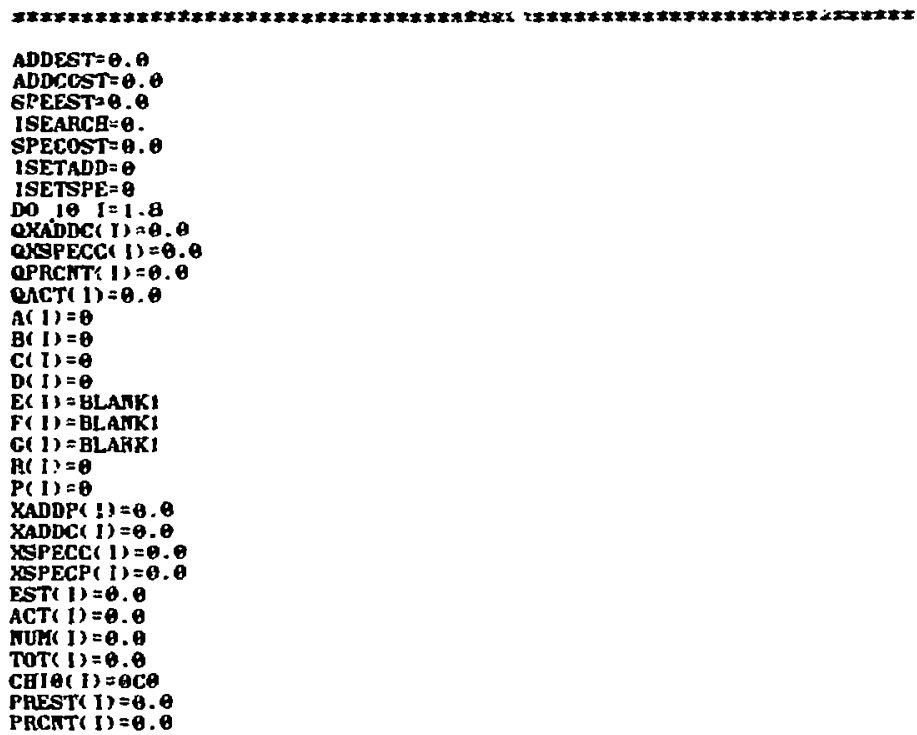


PRTUT: $11-9,0$

10 CONTINLE

Cus $11=0$.

$\cos 11=0.0$

$\cos (14=0.0$

$\mathrm{C}$
$\mathrm{C}$
$\mathrm{C}$
$\mathrm{C}$
$\mathrm{C}$
$\mathrm{C}$
$\mathrm{C}$
$\mathrm{C}$
$\mathrm{C}$
$\mathrm{C}$
$\mathrm{C}$
$\mathrm{C}$
$\mathrm{C}$
$\mathrm{C}$
$\mathrm{C}$
$\mathrm{C}$
$\mathrm{C}$
$\mathrm{C}$
$\mathrm{C}$

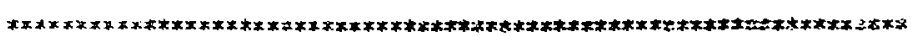

INDEX $=0$

YlRST PASS TRAU DATA TO OBTAIN SUPPUKT

NBEX $=1$

FRACTION

ISPEC =

I ADD $=1$

ISUM $=1$

I.IST $=1$

DATA rOR SUPPORT

SUMMARY OF SPECI AL EXMIIIATIONS

SUMMARY OF ADDITIONAL EXAMINATIOTS

SUMMAHY OF EXAHIMAT 1OFS

COMPLETE LISTING OF EACH FUEL PIF

COMPLETE LISTING OF EACH FUEL PIN

PRODÜCES SUMUARY OF SPECIAL AND ADDITIO

EXAMINATIONS, AND PINS COMPLETFD DURING

IANU $=1$

PRODUCES SUTMARY

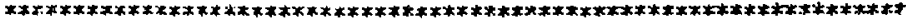

ISPEC $=0$

$I \mathrm{ADD}=\mathbf{0}$

ISUM $=\theta$

LIST $=0$

IQUART $=6$

I $A N N=0$

$1 \mathrm{CMB} 1=0$

LCKB II

LCMB 140

$\mathbf{c}$
$\mathbf{C}$
$\mathrm{C}$
$\mathrm{c}$
$\mathrm{C}$
$\mathrm{C}$

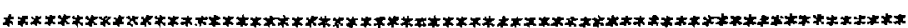

READS III CONTROL CARDS

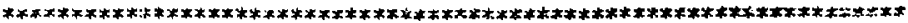

20 REA $(1,1456)$ ADATA

IF (EGF, 1) \$0,30

36 cont inue

IF (ADATAI 1). EQ.CKB 1) LCIB $1=1$

I5 (ADATA I), EQ. CMB I I) LCHBi $1=1$

IF (ADATAC 1). EQ. CMB 14) LCHB 14=1

IF ( ADATAC I , EQ. LISTDAT) GO TO BIO

IF (ADATAS I), EQ. FULi) LIST=1

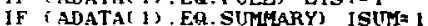

IF (ADATAC 1 ) EQ. ADD) IAND=

IF (ADATA( 1$)$. EQ. SPEC) ISPEC=

IF (ADATAC i), EQ. QUARTER IQUART $=1$

IF ( ADATAC i) EQ. ANMUAL IARN=1

$\mathrm{C}$
$\mathrm{C}$
$\mathbf{C}$
$\mathrm{C}$
$\mathrm{C}$
$\mathrm{C}$
$\mathrm{C}$
$\mathrm{C}$
$\mathrm{c}$
$\mathrm{C}$
$\mathrm{C}$
$\mathrm{C}$

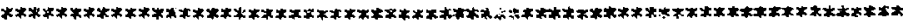

ISUM $=1$ GEKFRATEE ONLY TEE SUMHARY DATA, ROT A FILI. LISTING

LIST $=1$ COMPLFTE $i$. ISTING WITH SUTMATY DATA

ISPEC $=1$ L.ISTS ONL.Y THE SPECIAL EXAYINATIONS

IADD $=1$ LISTS GNLY THE ADDITIONAL EXAMINATIUNS

if ISPEC $=1$ AND IADQ $=1$ PRODUCES COMBIHATIC: IITTIRC OF

SPECIAL AIT ANDITIOTAL EXATINATIONS

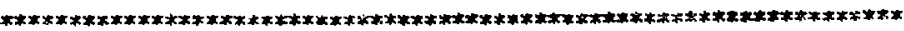

IF (ADATAC 1).EQ.START) DECODE (10,1460,ADATAC 2) i

IF (ADATAC 1). EQ. LAST) DECODE (10, i460, ABATA(2)) B

IF (ADATAR 1). EQ. F ISCAL) DECODE $(10,1460$, ADATA( 2) ) C

IF (ADATAL I) EO CHITS) DECODE (10. 460 , ADATAC

IF (ADATA (1).EQ.CBITS) DECODE ( 16, 1470,ADATA(3) TOTCHIT

if (ADATAI 1 ) . Fo. OSTART) DECODE $(16,1460$, ADATAC 2 ) ) P

IF (ADATA (1) . EQ. QEND) DECODE $(19,1460$, ADATAK 2$)$ )

CO $\mathrm{TO} 28$

49 CONT INUE

IF ( IA AT FO I) GO TO 50

if ( IADD, EQ. I) WHITE $(21,1500)$

if (ISPEC. Fa. 1) WRITF $(22,1498$ )

sa corTIRUt

FYERP $=1970+C(1)$

IF ( IADD.EQ. 1. AND. JANN. EQ. I) WRITE (21.8066) FYEAR

IF (ISPEC, EQ. I . AND. IANA. EO. 1) WRITE (22, 2070) FYEHA

if (IADD. EA. 1 . AND. IQUART. EQ. 1) WAITE (28, 1900)

iF (ISPEC. EO 1. ANT. IOUART. FA. 1) WRITE (29, 1910)

$D A T 29=A(2) * 10900 \theta+A(3) * 1000 \theta+A(4) * 1090+A(5) * 10 \theta+A(6) * 10+A(7)$

$D A T F I=B(1) * 10090 \theta+B(2) * 18000+B(3) * 1000+B(4) * 100+B(5) * 10+B(6)$

NCEI TQ $=\mathrm{D}(1)$

DAYST=Q $\theta$

AMTTOT 6.6 


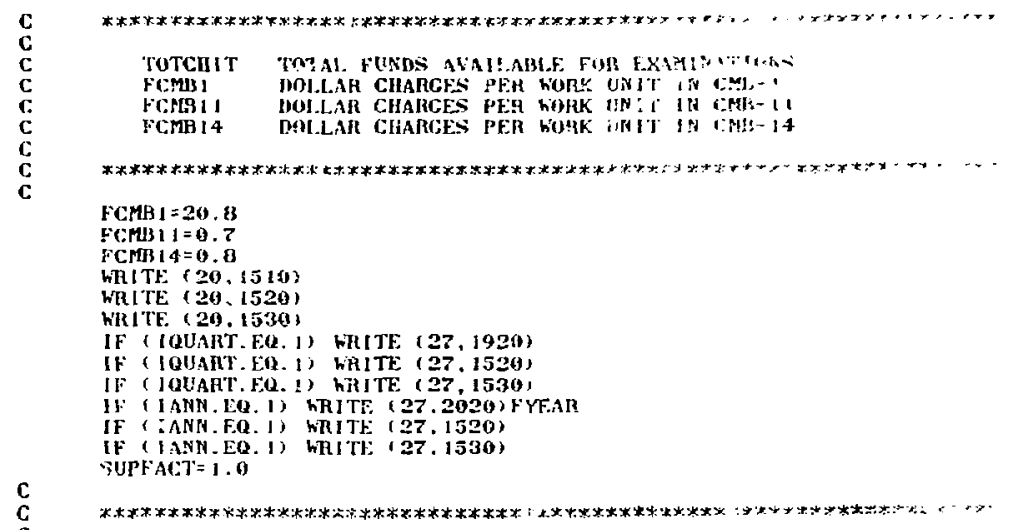

READS IN FXIPHIMENTER IDENTIFICATION, CODE WOHY, DOLLARS IR GAIT C $\quad$ C

Do To $1=1$, NCIIITS

JUEAD $(1,1540 ; \mathrm{FC}(1), \mathrm{F}(1), \mathrm{C}(1), \mathrm{CO}) \mathrm{E}(\mathrm{I}), \mathrm{CH} 1 \mathrm{~T}(1)$

If (EOF, 1$) 80,60$

60 CONTINIJ

70 CONTISIS

80 CONTINUE

C
C
C
C
C
C
C
C

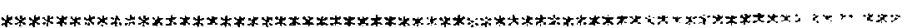

LFY LAST FISEAL YERR

MFY MONEY FISCAL YEAR - PRFSENT YEAR

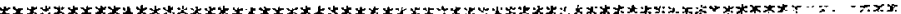

RFAD (1.118O) ICOPY

IF (FOF I) 90,100

$90 \quad I C G P Y=1$

190 CONTINUE

FF.AD $(1,15 \overline{5} 014 \mathrm{TITLE}(L), L=1, \overline{6})$

IF (EOF, 1) 110,130

110 hTLTF: $(2,1160)$

120 CONTINUE

AMTTOT $=0.0$

CAL.L. DATE. (MMDDYY)

DECODE ( 10,1190, PRDDYY) IMTH, IDAY, IY

L.F $Y=$ F YEAR- I

MF $Y=F Y E: A I R$

N12=13(1)*10+B(2)

M34 $=B(3) *(0+B \div 4)$

$M 6=B(5) * 10+13(6)$

$N 12=A(2) * 10+A(j)$

N34 $=1(4) * 10 \div A(5)$

$N 5 B=A(6) * 10+A(?$,

QS $12=\mathrm{P}: 1\} * 3\left(0+\mathrm{P}_{1} \mathrm{P}\right)$

QS3 $34=P(3) * 10+P(4)$

$0 \times 56=P(5) * 10+P(6)$

(2) $12=R(1) * 10+R(2)$

QF: $12=R(1) *(1)+R(2)$

QE56 $=R(5) * 10+11(6)$

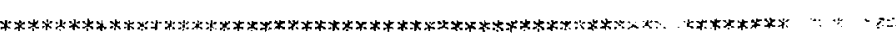

M12 PHESENT NONTH

M34 PIFEENT DAY

MJ6 PILSENT YEAR

FOR COMPUTING SUPPORT FRACTJONS

FI? MONTH OF STALTING DATE

N12 MONTH OF STALTING DAT
N34 DAY OF STARTING DATE

N56 YEAR OY STARTING DATE

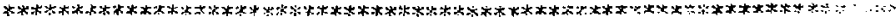

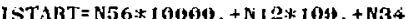

IEND $=M 56 * 10000+N 12 * 100+M 34$

IEND $=$ TIS
DAYS $=0$

QDAYS $=0$

IF (N12.GT.Z) DAYS= DAYS+31

IF (N12.(GT.B) DAYSi= BAYS+3)

IF iN12.GT.9) D.AYS= D.AYS+30

IF ( N $12 . G T .10)$ DAYS= D.YYS+31

IF (NI2.GT. II) DAYSE $[1 .+Y S+30$ 


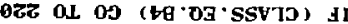

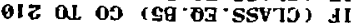

ooz ol 03 (Gn.og-SStiJ) AI

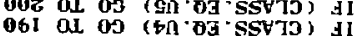

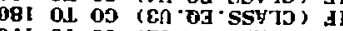

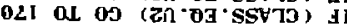

091 OL 09 (In'03'SSH7) d

*F

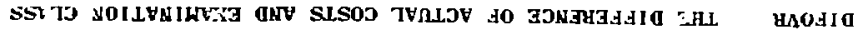

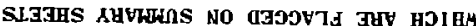

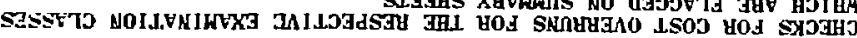

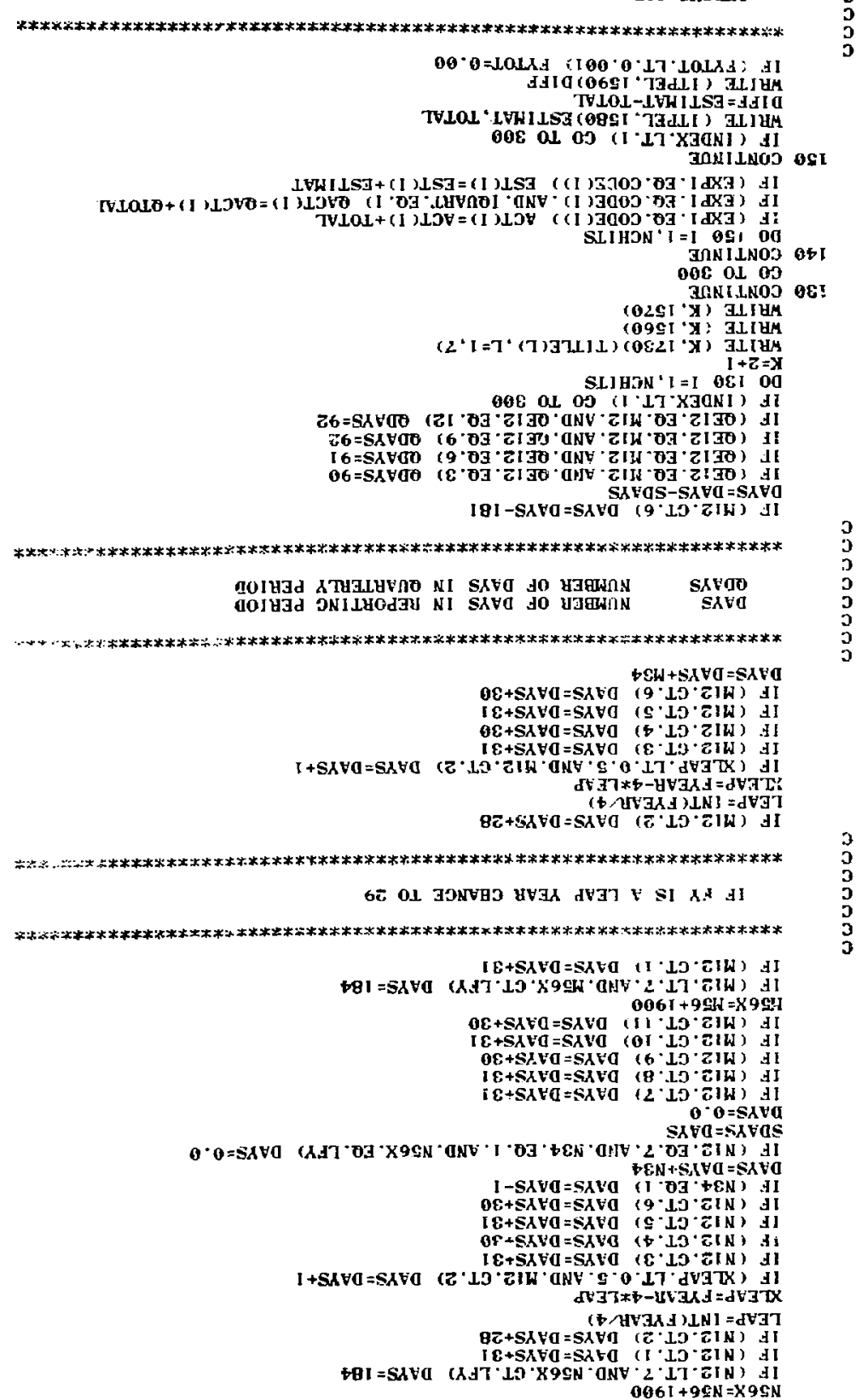


IF (CLABS. 20. B3) 0 no 230

IP (CLASS. 20. B2) CO T0 240

IF (CLABS.EA.BI) $60 \mathrm{T0} 250$

$$
\text { G) } 2026
$$

160 CorTI

DIFOVR= TOTCBT-CLASBIT

IF (CLABS. DO. U1.AND. TOTCST. GT.CLASSID.AND. ICONP. Ra. I) IRITE (20.12 110 ) NULX, EXP1, EXP2, EXP9, CLASB, TOTA1, TOTEST, DJFOVh

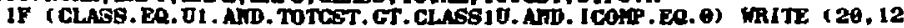
109) NUTK, EXP 1, EXP2, EXP3, CLASB, TATA1, TOTCET, D IFOVA

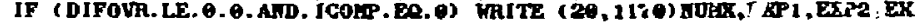
1P3, CLASB, TOTAL, TOTCST

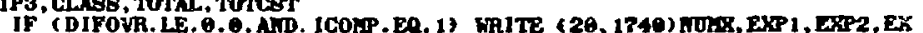
1 P3, CLASB, TUTAL, TOTCET

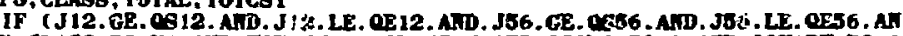

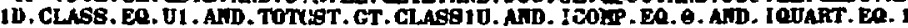
a) WRTE (22, 1200) KU, EXP1, EXP2, EXP3, GLAS, TOTAL, TOTEST, DIPOVA

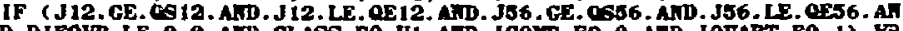

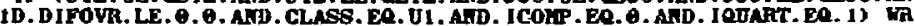

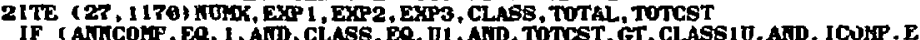
(4. ф) WRITE (27, 1209) NUIK, EXP 1, EXP2, EXP3, CLASB, TOTAL, TOTCST, DIFOVA

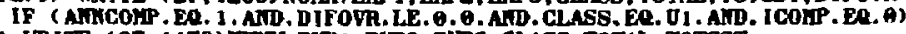
1 WRITE (27, 1 178) NUIX, EXP I, EXP2, EXP3, CLASS, TOTAL, TOTCBT

co To 270

170 CorTing

DIFOVR = TOMCST-CLASSQ"

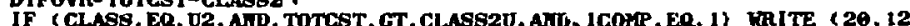

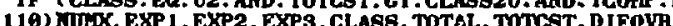

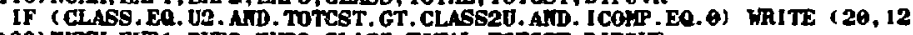
106) NUTK, EXP I, EXP2, EXP3, CLABS, TOTAC, TOTCST, D IFOVR

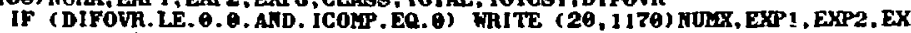
IP3, CLABS, TOTAL. TITCBT

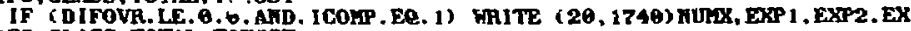
IPB, CLASE, TOTAL, TOTCST

IF (J12.GE. OS12. AND. J12.LE. OE12, AND.J66.GE. OSJ6. .WJ. J56. LE. OE56. AN

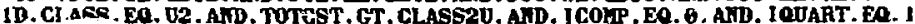
2) WRITE ( 27,1200$)$ NUTK, EXP 1, EXP2, DXP3, CLASS, TOTAL, TOTCST, DIFOVA

IF ( J12.GE. OS12. AND.J12.LE. OE12.AND.J56.GE. OSJ6.AND.J56. LE. OEJ6. AT

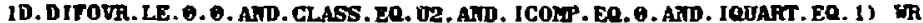
2ITE (27, 1 170) NUTX, EXP 1, EXP2, EXP3, CLASS, TOTAL, TONCST

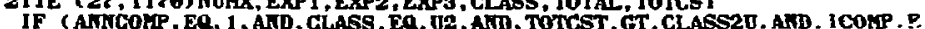

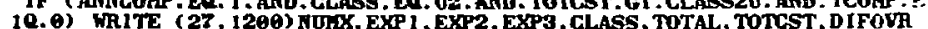

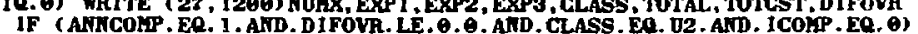

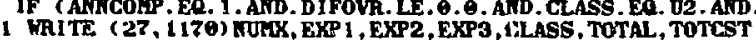

Go TO 270

189 CONTIKUE

D IFOVR= TOTCST-CLASSAU

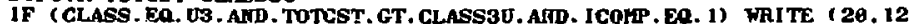
1 10) NUYX, EXP1, EXP2, EXP3, CLABS, TOTAL, TOTCST, DIFOVH

10 MUK, EXP1, EXP2, EXP3, CLABs, TOTAL, TOTCST, Dr. IF (CLASS . EO. U3. AND.TOTCST. GT. CLASS3U. AND. ICOFP.

IF (DIFOVR. LE, 0.0. AND. ICONP. EO, 0 ) WRITE (20,1170) NUNX, EXPI, EXP2, EX IP3, CLASS, TOTAL, TOTCET

IF (DIFOVR. LE. O. . AND. ICOYP, EQ. 1) WRITE (20, 1Z40) ROTX, EXP1, EXP2, EX IP3, CLABS, TOTAL, TOTLST

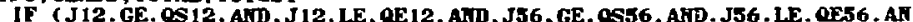
ID. ClASS EQ UB AND TOTCST GT CLASGBU. AID ICOMP EO. A. AND IOUAHT. ER. I 2) WAITE (27, 1200) FUTX, JXP 1, EXP2, EXP3, CLASS, TUTAL TOTCST, DIFOUR

2) WAITE (27, 1200) KUTX, EXP1, EXP2, EXP3, CLASS, TUTAL, TOTCST, DIFOVR

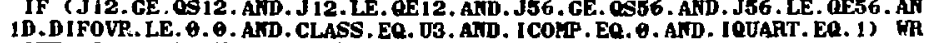
$2 I T E(27,1170)$ NUHK. EXP 1, EXP2, EXP3, CLASS, TOTAL, TOTCST

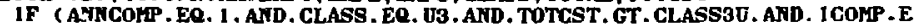
10. 0) WTITE (27, 1200) NOTX, EXP1 , EXP2, EXP3, CLASS, TOTAL, TOTCST, D1FOVA

IF ( ANNCOKP. EQ. 1 AND. DIFOVR. LE. 0.6. AND. GLASS. EO. US, AND. ICOHP . EQ . 6 )

1 WRITE (27, 1170) NULX, EXP 1, FXP2, EXP3, CLASS, TOTAL, TOTCST

WRITE $(2$

190 CO TO 270

DIFOVR=TOTCST-CLASSAU

IF (CLASS. ER. U4. AND. TOTCST. GT. CLASS40. AND. ICOHP. EQ, 1) WRITE (2a, 12 119) NOIX, EXP 1, EXP2, EXP3, CLASS, TOTAL, TOTCST, DIFOVR

IF (CLASS. EO. U4 AND. TOTCST. GT. CLASS4U. AND. ICOYP. EQ. 100) NUTX, EXP 1, EXP2, EXP3 CLASG, T0TAL TOTCST, DIFOVR

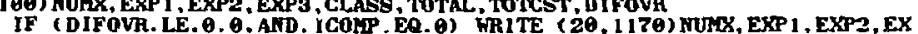
IP3, CLASS, TOTAI, TOTCST

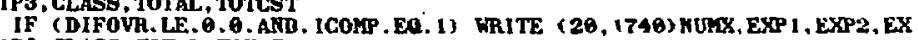
IP3, CLASS, TOTAL. TOTCST

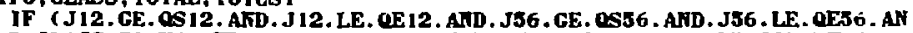
1D. CLASS. EQ. U4, AND. TOTCST, GT. CLASS4U. AND. I CONP. EQ. B. AND. IQUART. EQ. 2) WR 1TE (27, 1200) NULX, EXP 1, EXP2, EXP3, CLASS, TOTAL, TOTCST, D1FOVR

IF ( J 12. GE. OS12. AND. J 12 . LE. OE12. AND. J56. GE. OS56. AND. J56. LE. OEJ6. AN 1D. DIFOVA. LE. 6.6. AND. CLASS. EQ. V\$. AND. ICOHP. EQ. G. AND. 1ODART. EQ. 1) Wh 2 ITE ( 27, 1170$)$ HUIX, EXP 1, EXP2, EXP3, CLASS, TOTAL, TOTCST

IF ( ANTCONP. EQ. 1 AND. CLASS. ER. U4. AND. TOTCST, GT. CLASS4U. AND. ICOHP. E 10.0) WRITE (27, 1200) NUHX, EXP1, EXP2, EXP3, CLASS, TOTAL, TOTCST, D IFOVA

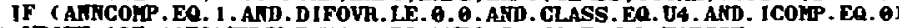

1 WR ITE ( 27, 1170 ) FUNX, EXP I, EXP2, EXP3, CLASS, TOTAL, TOTCST

co TO 270 
20 CONTINUE

DIFOVA= TOTCST - CLAESSU

if (CLASS EQ. US. AND. TOTCST, CT CLASS5U. AND. ICOKP. EU. I) WRITE (20,12 1163 MUNX, EXP 1, EXP2. EXP3, CLASS, TOTAL, TOTCST, DIFOVR

IF (CLASS. EQ. Ub. AND TOTCST.GT.CLASS5U.AND. ICOHP. EO. 6) IRITE (20, 12 100) NUMK, EXP I , EXP2, EXP3, CLASS, TOTAL, TOTCST, D IFOVH

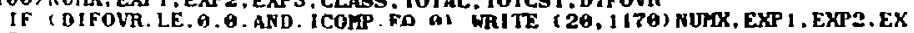
IP3. Class, TOTAL. TOTCST

IF (DIFOVR LE. O. O. AND. ICOMP. EQ. I) WAITE (20,1740) NUPX, EXP 1.EXP2. EX IP3, CLASS, TOTAL. TOTCST

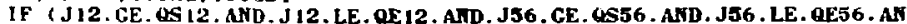
10. CLASS. EQ. US AND. MTCST. GT. CLASSSU. AND. ICOHP. EQ. O. AND. IQUART. EQ. I 2) WAITE $(27,1260)$ NINAX, EXP I , EXP2, EXP3, CLASS, TOTAL. TOTCST, D IFOVR

IF I J 12.GE. OS 12. AND.Ji2. LE. OE12. AND.J66.GL. QS56 AND.J56. LE. QEJ6. AN

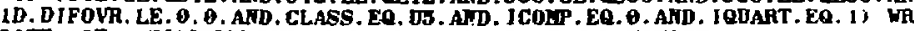
21TE (27, 1170$)$ NUNX, EXP1, EXP2, EXP3, CLASS, TOTAL, TOTCST

IF ( ANRCOMP . EQ . 1 AND . CLASS, EQ. US AND. TOTCST. GT. CLASSEU. AND. ICONP. E 10. $)$ ) WH I TE $(27,1200)$ NUIX, EXP 1, EXP2, EXP3, CLASS, TOTAL, TOTCST, D I FOVR

IF ( ANNCONP. EQ. I AND . D IFOVR. LE. G G AND. Cl.ASS, EQ. US . AFD. ICOUP. EQ. 6 ) 1 HRITE $(27,1170)$ NUNX, EXP 1, EXP2, EXP3, CI ASS, TOTAL. TOTCST

GO TO 278

210 CONT TO 27

DIFOVR= TOTCST - CLASSBB

IF (CLASS. EQ. B5. AND. TOTCST. GT. CLASSEB. AND. ICOHP. EQ. I) WRITE (20. 12

(10) NUMX, EXP 1, EXP2, EXP3, CLASS, TOTAL, TOTCST, DIFOVR

IF (CLASS. EQ. B5. AND . TOTCST, GT CLASS5 B. AND. ICOHP. EQ. $\theta)$ WRITE (20, I2 $190)$ NUNX, EXP1, FXP2 EXPA, CLASS 'MTAL, TOTCST, DIFOVR

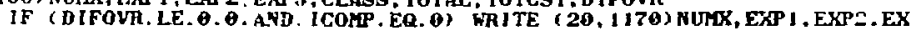

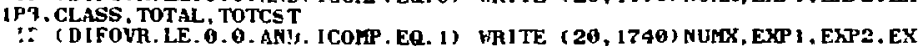
IP3, CLASS, TOTAL, TOTCST

IF (J12. GE. QS12. AND.J12. LE. QE 12. AND. J56. GE. QS56. AND. J56 . LE. OF56. AN

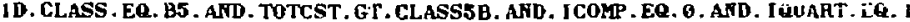
2) WRITE $(2 \overline{6}, 1200)$ NUNX, EXP 1, EXi'2, EXP3, CLASS, TOTAL, TOTCST, D IFUVK

IF (J12, GE. OSI2. AND.J 12. LE. OE 12, AITD.J56.GE. OS56. AND. J56. LE. HE56. AN

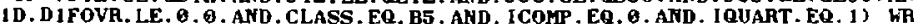
2 IT2 (27, 1, .70) NUMX, EXP 1, EXP2, EXP3, CLASS, TOTAL, TOTCST

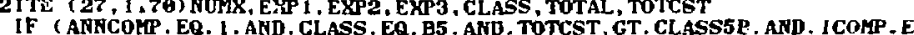

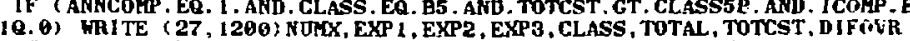

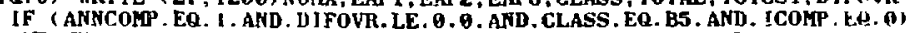
1 NRITE (27, 1170) NUEK, EXP1, EXP2, EXP3, CLASS, TOTAL. TOTCST

Co $\mathrm{T0} 270$

220 COHTINUE

DIFOVR= TOTEST - CLASSAB

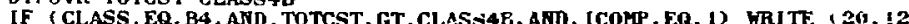

116 ) NURX, EXP1. EXP2, EXP3, CLASE, TOTAL, TOTCST, DIFOVR

If (CLASS. EQ. B4. AND. TO'CST. GT.CLASS4B. AND. ICOHP. EQ.0) WRITE (20.12 (90) NUMX, EXP I , EXP2, E.XP3, CLASS, TOTAL. TOTCST. DIFOVF

IF (D JFOVA.LE. O.0. AFD. ICOPP. EQ.0) HRITE $(20,1170)$ NUMX, EXP I, EXP2, EY 1P3, CLASS, TOTAL, TOTCST

IF (D1FOVH.LE. O.6. AND. ICOMP. EQ. 1) WR1TE (20,1740) NURE, EXP1,EXP2, EX 1 P3. C.LASS TOTAL. TOTAST

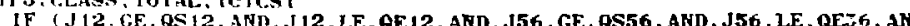

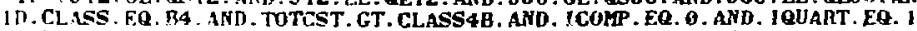

2) HTITE (2T, 1200 ) NUHX. EXP 1, EXP2, EXP3, CLASS, TOTAL, TOTCST, D IFOVR

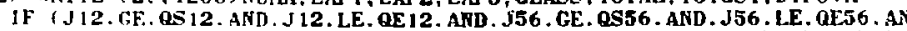
ID. DIFOVR. LE. O . O. AND. CLASS. EO, B4. AND. ICOMP. EQ, O. AND. IQUART. EO. S; ST 2 ITE (27, 1170) NITM, EXP1, EXP2, EXP3, CLASS, TOTAL, TOTCST

IF I ANNCOMP . FQ. 1 AND. CLASS . EQ. B4 . AND. TOTCST. GT . CLASS4B. AND. ICOMP. E 10. B) WRITE (27, 1206) NUMX, EXP 1, EXP2, EXP3, CLASS, TOTAL, TOTCST, D IFOUA

IF , ANMCOMP . EQ 1 . AND. DIFOVR. LE. O. O. AND. CLASS. EQ. BA. AND. 1 COMP. ER.O

1 WR ITF $(27,1170)$ NUPX, F,XP1, EXP2, EXP3, CLASS, TOTAL, TOTCST

G) TO $27 \%$

230 CONTINIS

D I FOVR $=$ TOTCST - CLASSBA

IF ( CLASS. EQ. B3. AND. TOTCST, GT. CLASS3B. AND. ICOMP. EQ. 1$)$ WRITE (20, 12 116 ) NUMX, EXP 1 , EXP2, EXP3, CLASS, TOTAL, TOTCST, D I FOVH

IF (CLASS EQ B3. AND TOTCST GT. CLASSBB. AND. ICOMP EO. O) WRITE $(20,12$ 100) NUMK, EXP1, EXP2, EXP3, CLASS, TOTAL, TOTCST, D IFOVH

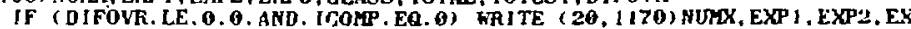
IF ( DIFOVR. LE, O. O. AND
1 P3, CLASS, TOTAL, TOTCST

IF (DIFOVR. LE. O.O.AND. (COMP. EQ. 1) WRITE $(20,1740)$ NURX, EXP 1 . EXP2. EX IP3, ClASS, TOTAL, TOTCST

IF ( J 12.GE. OS 12. AND. J 12. LE. OE.12. AND. J56. GE. OS56 . AND. J56 . LE. QE A . AN ID. CLASS . EQ. B3 . AND. TOTCST. GT. CLASS3R. AND. ICOHP . EQ O . AND. IQUART , LA. I 2) HRITE ( 27,1290$)$ MTIR, EXP 1, EXP2, EXP3, CLASS, TOTAL, TOTCST, DIFOVR

IF (J12.GE. OS12. AND.J 12.LE. OE 12. AND. J56.GE. OS56. AND.J56. LE. OES6. AN ID. B IFOVR. LE. B. B. AND. CLASS. EQ. B3. AND. ICONP.EQ. B. AND. IQUART. CO. 1 " WR 2 ITE $(27,1170)$ NUNX, EXP 1, EXP2, EXP3, CLASS, TOTAL, TOTCST

If (ANNCOHP . EQ - I AND . CLASS. EQ. B3 . AND. TOTCST.GT. CLASSBB. AND. ICUNP. E 10.0) HRI TE (27, 1206, NUREX, EXP 1 EXP2, EXP3, CLASS, TOTAL, TOTCST, D I FOVR

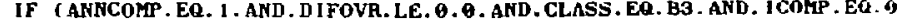

1 WRI'TE $(27,1170)$ NUTX. EXP 1, EXP2, EXP3, CLASS, TOTAL. 'TUICS'

1 HRITE 127

240 CONTINUE

DIFOVR= TOTCST - CLASS2B

IF (CLASS. EQ. B2. AND. TOTCST. GT. CLASS2B. AFD. ICOMP. EQ. 1) WRITF, (20,12 1 10) NUTX, EXP 1 , EXP2, EXP3, CLASS, TOTAL, TOTCST, DIFOVR

IF (CLASS. EQ. D2. AND. TOTCST. GT. CLASS2B.AND. ICOHP. EO. 0) WRITE 12.12 100) NUHX, EXP 1, EXP2, EXP3 CLASS, TOTAL, TOTCST, DIFOVR

IF (DIFOVR. LE. O.O. AFD. ICOHP, EQ.0) IRITE $(20,1170)$ NUMX, EXP 1,E:T2, EX

1P3. CLASS. TOTAL. TOTCST 


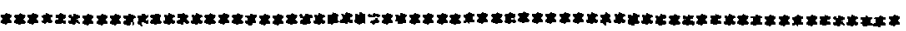

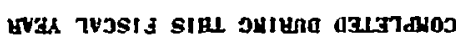
a:157dhos

arextofo3 Jon

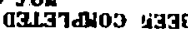

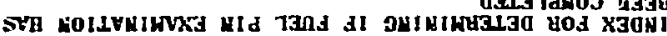

$$
\begin{aligned}
& I \text { = DHOJNNV } \\
& \begin{array}{l}
1=\text { dHODI } \\
1=\text { dU031 }
\end{array} \\
& \text { d4031 }
\end{aligned}
$$

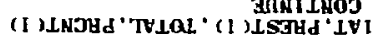

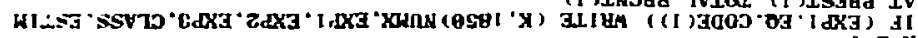

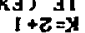

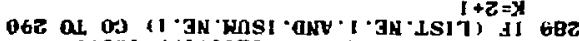

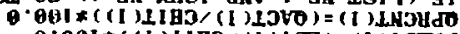

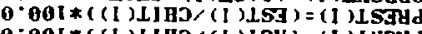

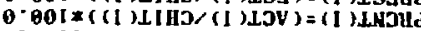

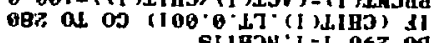
SLIEDN $I=1$ O6Z OC SLSOJ

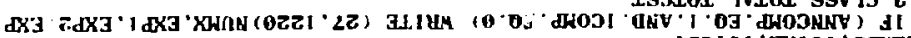
LSDLAL TVJAL SSTIZ

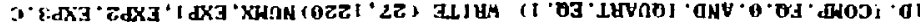

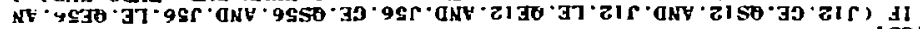
N LSI iol'THLW'

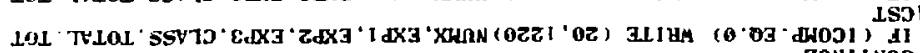
InHILNDJ $69 \mathrm{E}$ 028 al. od

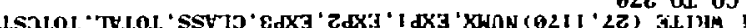

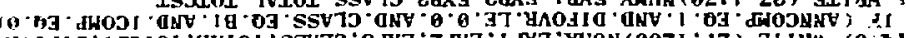

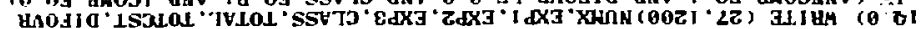

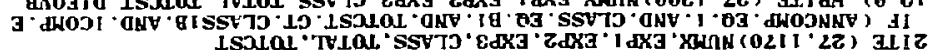

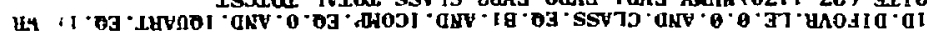

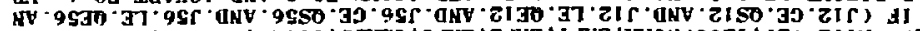

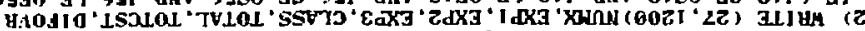

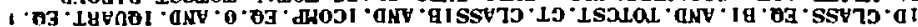

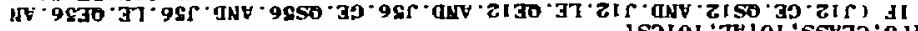
ASDLOL "TVLLL 'SSVTJ' Ed I

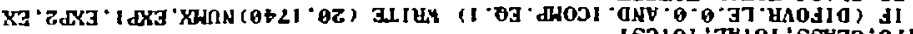
X3'

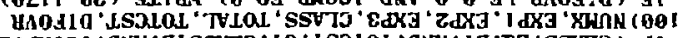

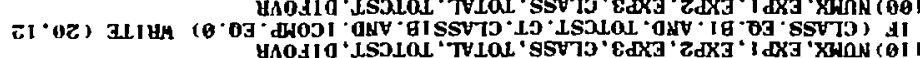

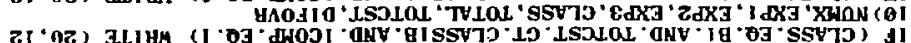

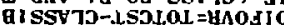
InNILNOS osz

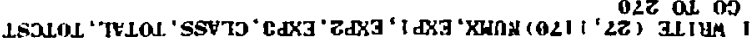

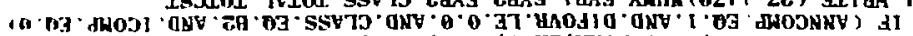

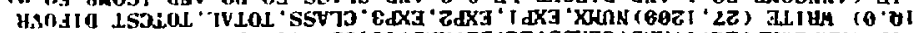

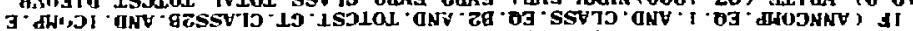

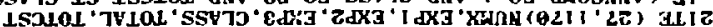

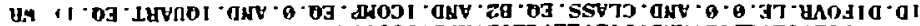

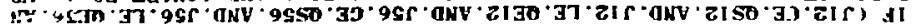

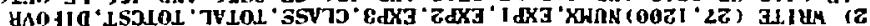

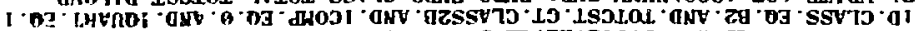

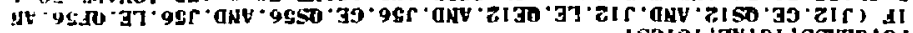

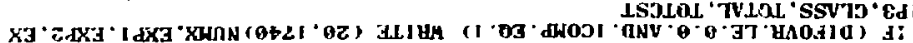




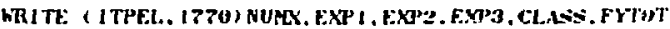

WRITE (ITPEL.. 1780)

DO $330 \quad i=1$. NCHITS

IF (EXP I. EQ. CODEA 1 , NUY $11=$ stIM 1 ) +1

330 CONTINUE

340 CONTINUE.

TOTAL=0.0

ESTIMAT $=0.0$

OCP IE $=0.0$

QTOTIL $=0$.

ANMCOMP $=0$

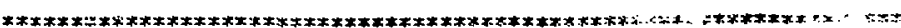

CHECK FOUR DICIT CODE FOR PROLRAM CONTHOH.

TYPE I AND TYPER DESCRIPTION PARAMETERS

IDATECP DATE PIE COMPLETED

IDATERQ DATE PIE REQUESTED

IDATERP DATE PIE REPGRTED

WRKUNT WOHK UNIT VALUES ASEIGNED TO EACI EXMMIJATIOA

IGIPP GROUP PERFORING EXAMINATION

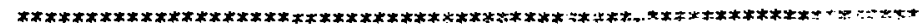

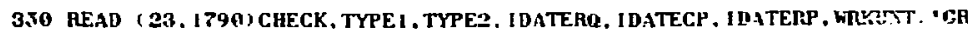
$1 \mathrm{P}$

IF (ENDFILE 2A) 450.360

360 CONTINUE

IF (CHECK. EU. ASTERA) ISETADD $=0$

IF ( .HECK. EQ. ASTER4) ISETSPE=0

IF (CiECK. EQ. ASTER4) CO TO 140

IF (CHECK. EO. ASTER3) ISETADD $=1$

IF (CHECK. EQ. ASTERA) ISETSPE $=0$

IF (CEECK. EQ. ASTERO) ISETSPE=

IF (CHECK. EQ.ASTERO) ISETADD $=0$

IF (INDEX. LT. I) GO TO 370

IF (CEECK. EQ. ASTERB) WRITE (ITPEL, 16 I0)

IF (CKECK. EQ. ASTEI3) CO TO 350

IF (CHECK. EO. ASTER2) WAITE ( ITPEL, 1600)

IF (CHECK. EQ. ASTER2) GO TO 350

IF (CEECK.EQ. NDT. AND TYPE I EO CAP) FRITE (ITPEL, 1800)

IF (CHECK. EQ. NDT. AND. TYPE I.EQ.CAP) GO TO 350

IF (CHECK. EQ. NDT, AND. TYPE l.EQ.PIN) WRITE (ITPEL, 1810)

IF (CHECK. EQ. NDT. AND. TYPEI.EQ. PIN) GO TO 350

IF (CHECK. EO. DEST) WRITE (1TPEL, 1820)

IF (CHECK. EQ. DEST) Go TO 35?

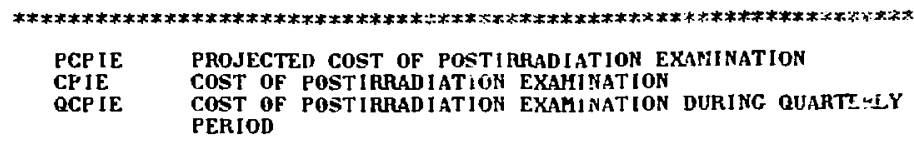

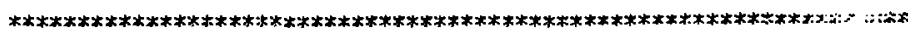
SELECTS WHICH GROUP FACTOR WILL BE USED TO COMPITTE CHARGFS

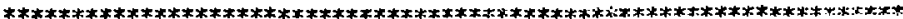

IF ( ISETADD. NE. 1, AND, I DATERQ. GT . O. AND. IDATECP. LT. 1 ) ICOMP= 1

IF (IGRP. EQ. 1) FACTOR = FCMB I

If ( IGRP. EO. I1) FACTOR= FCIB 1 )

IF (IGRP. EQ. 14) FACTOR=FCHB I4

IF ( IGRP, EO. O) FACTOR=0.0

If (IGRP. 60.99 ) 60 To 440

IF (IDATECP. LT. 1) Co TO 380

$112=1 D A T E C P / 10000$.

$134=I D A T E C P, 100 .-I 12 * 100$.

I56 = I DATECP-112*10000 - 134*100.

ISTEND $=156 * 10000+112 * 100+134$

ITRUE 12=0

ITAUE56=0

IF (I12. CE. OS I2. AND. 1 12. LE. OE12. AND. I 12.GT. JI2. AND. ISETADD. NE. I . AN

1D. IQUART. EQ. 11 ITRUE $12=1$

IF C I56. GE. OS56. AND. I56. LE. OEJ6 . AND. 156, GT. J56. AND. ISETADD . NE. I . AN

1D. IOUART. EQ. I) ITHUE56 $=1$ 


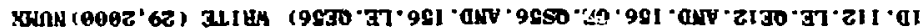

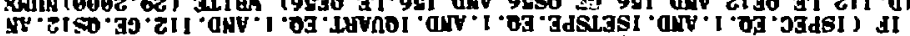

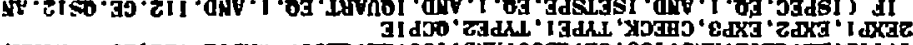

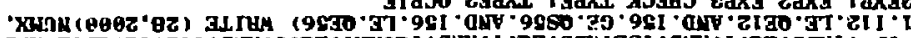

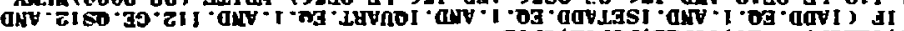

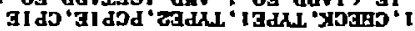
EdX3 '

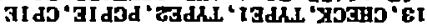

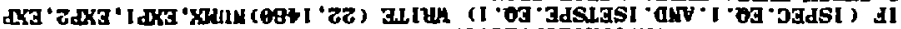

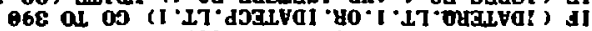

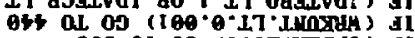
gge of oj (1. IT X3ani ) dI LJV.AdกS*

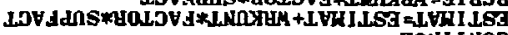

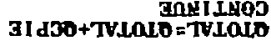

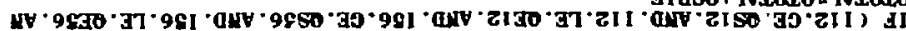

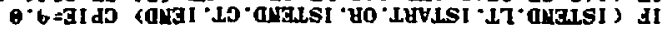

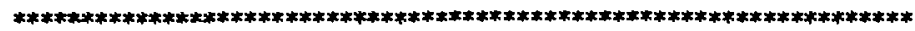

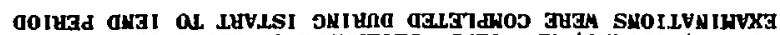

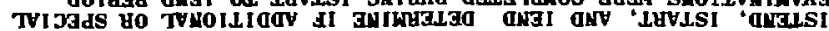

******************************************************************************

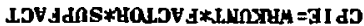

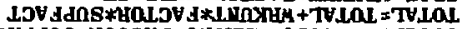

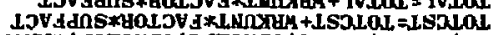

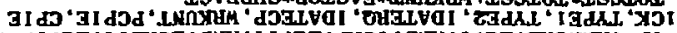

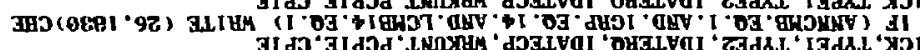

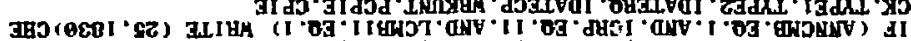

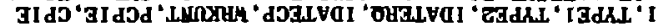

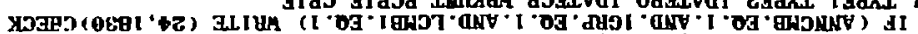

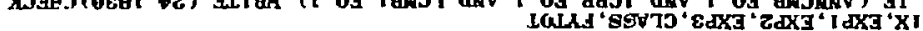

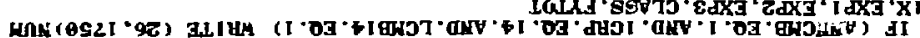

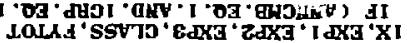

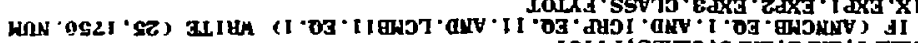

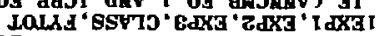

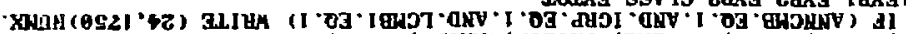

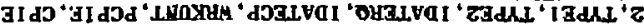

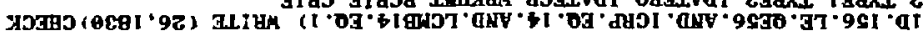

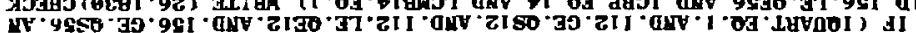

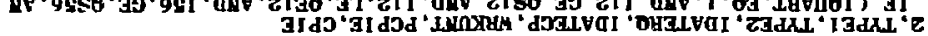

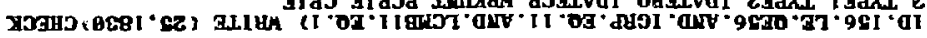

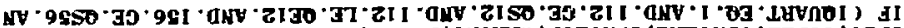

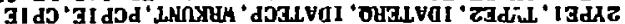

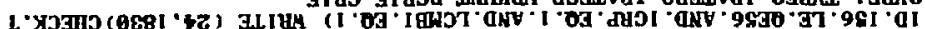

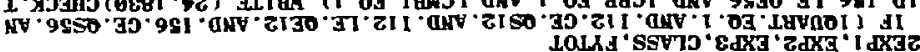

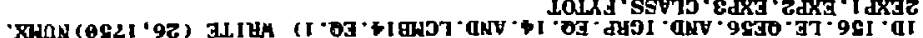

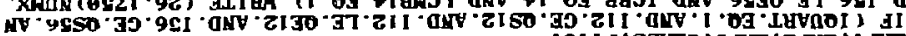

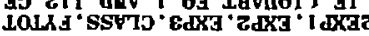

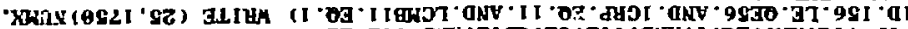

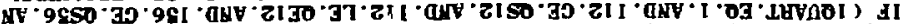

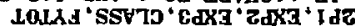

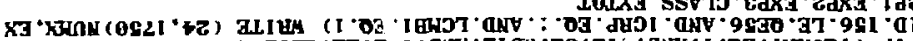

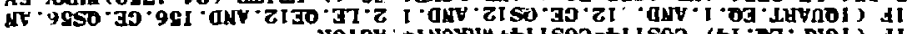

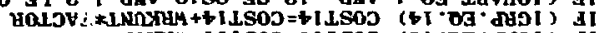

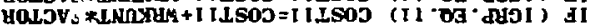

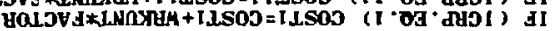

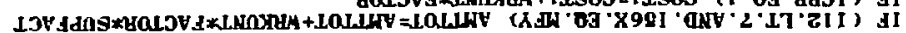

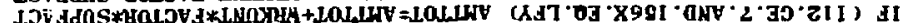

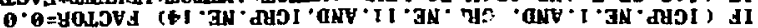

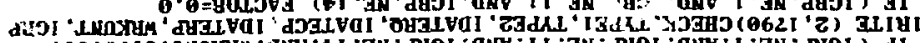

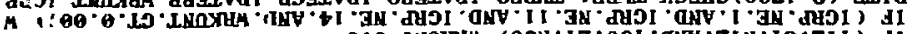

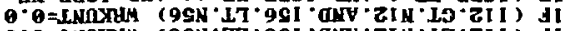

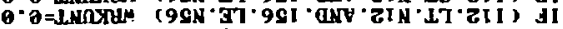

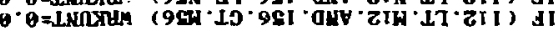

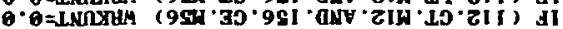

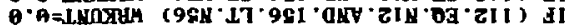
$991+086 \mathrm{I}=\mathrm{X991}$

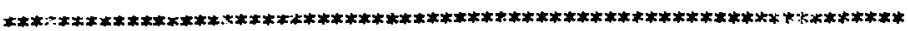

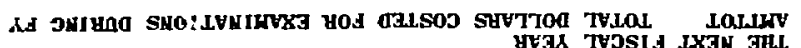

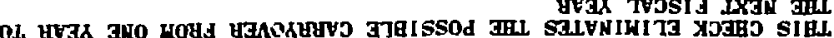

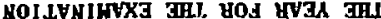

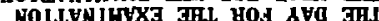

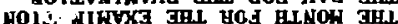

$99 I$

76

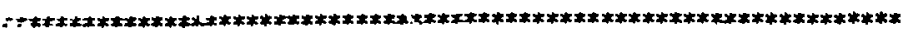

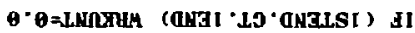

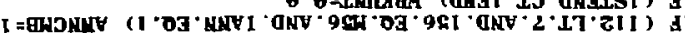

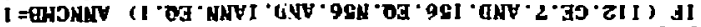

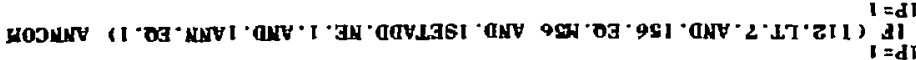

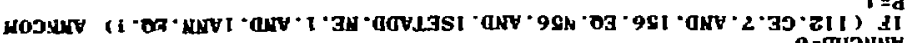
$0=$ IIHSWUV

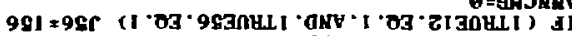

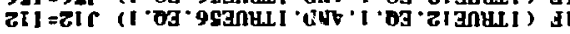




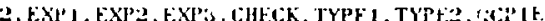

390 CONTINUE:

IF (ISPEC. NE. I.OR. ISFTSPE. NE. 1) GO TH 410

bo $4001=?$. NEHITS

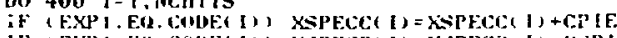

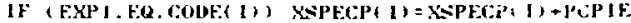

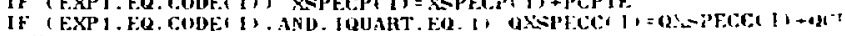

400 CURTINUR

410 CONiTUE

IF (IADD. NE. I, OR. ISFTADD. NE. 1 , 60 TO $4: 30$

Do $4201=1$. NCHiTs

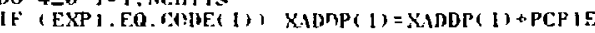

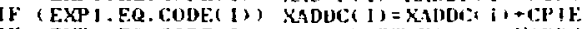

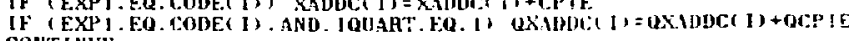

420 CONT INUF

430 ConTI YUE

449 CONTINUE

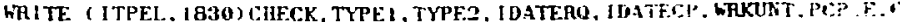

IPIE:

IF', IGRP, EQ O) WTITE I ITPEL I840

60 TO 350

450 CONTINUt

INDEX $=[$ INDEX+1

IF ( I NDEX. GT. I) GO TO 480

AMT $=0.0$

DO $460 \mathrm{I}=1$. NCHITS

$\mathrm{AMT}=\mathrm{AMT}+\mathrm{ACT} \mathrm{C}$,

460 CONTINUE

C
C
C
c

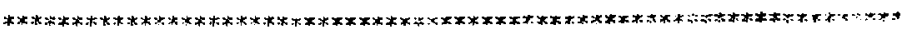

CONPUTES SUPPORT F ACTOR FON FINAL PASS THAU WATI SFTS

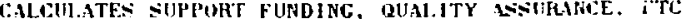

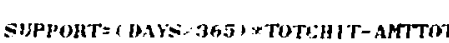

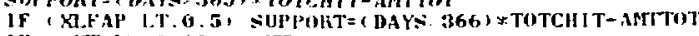

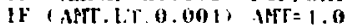

Do $4761=1$, NGHIIS

NUMI 1$)=0$

AC:T $(1)=0.0$

TOTI 1$)=0.9$

Torr $1,=0.6$

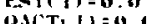

(2)

Dx.1B, $x=0.0$

QXsiptecc=0

470 CONTINHE

Tr:PIP $1=$ COST'

TE:MT' $1=$ COST I

TEMP $1+2=0$ CONT

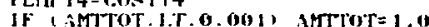

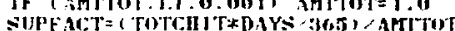

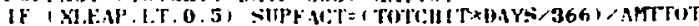

$K=0$

COSTI $=0.0$

cos' ! $1=0$. 1

COST $14=0.0$

HE'T NII $2: 3$

HFin' IN! 24

tif.h

ITE. $T$ INI 2.1

HFINI) 26

IPTTET'=

460 t:ONT I Nut

(ii) 49 II I $=1$. NE:H I T:

phisite $1,=0,0$

IF CHITI, I.T.

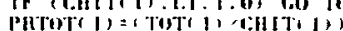

490 CONTINIF

ijt: $500 \quad i=1, a$

$K=1+2$

$1 .=1 \cdot 113$

C
C
C
c

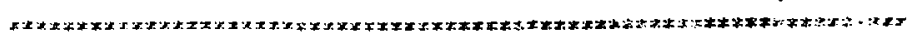

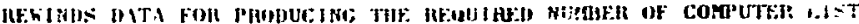

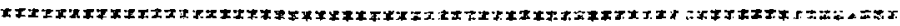

Man ria.: $k$

rint rll.E:

IFhIND $\mathrm{K}$

Jikind

sen cont isus:

riti fil.t: 21

titi f It.F.

ItFkIUI 2

IEHINU 2:

HES + II.F: 20 
REWIND 20

END FIJ.E 24

END FIIE 24

END FII.E 25

END F!LE 25

END FILE 25

FND FILE 26

END FILE 2

REWIND 27

ENI FILE 2B

REWIND 28

END FILE 29

REWIND 29

$\mathrm{L}=1$

$\mathrm{L}=1$ CONT IMOE

$\mathrm{L}=\mathrm{L}+\mathbf{1}$

WRJTE $(2,1270)$ ( TITLE $]$ ), $I=1,7)$, I HTH, IDAY, IYR

WAITE $(2,1620)$ HCH ITS

WRI TE $(2,1640)$

$\operatorname{COST} 1=$ TEMP

COST $11=T E M P 11$

$\cos T 14=$ TEMP 14

TOTGFP $=\operatorname{cosT} 1+\cos T 11+\cos T 14$

TRCNT $1=(\operatorname{Cos} 1 /$ TOTGRP $) * 100.0$

PRC 1 T 1 = ( COST $11 /$ TOTCRP $) * 100.0$

PRCHT $1=($ COST $11 /$ TOTCRP $) * 100.6$
PRCNT $14=($ COST $14 /$ TOTCPP $) * 100.0$

VRITE $(2,1650)$ SUPPORT, AMT, DAYS

IF ( IOUART. EQ. 1$)$ WRITE $(2,2010)$ ODAYS

TOT $1=0.0$

TOT $11=0.0$

TOT $14=0.0$

TOT $1=\operatorname{COST} 1 *$ SUPFACT

TOT $11=\operatorname{COST} 11$ ISUPF ACT

TOT $14=\operatorname{COST} 14 * S U P F A C T$

WHITE $(2,16 \pi 0)$

WHITE $(2,1700) \cos 11$, TOT 1, PRCNT 1

WHITE (2.17 10) COST 1 I TOT I I, PRCNT 1 ।

HAITE $(2,1729)$ COST!S, TOT 14, PRCNT 14

WHITE $(2,1419)$

WRITE ( 2,1230$)$

WRITE $(2,1240)$

IF (N34.GT.9) WRITE (2, I250) FYEAR, N12, H34, H36, HI 2, M34, H46

IF (N34.LT, 10) WRITE 12,1266$)$ FYEAR, N I2, N34, N56, M12, MB4, NG6

WRITE $(2,1870)$ FYEAR

DO $529 I=1 . N C B I T S$

iHC ()

520 CONT IRUE

WHI TE $(2,1670)$

WHITE $(2,1680)$

DO 530 1 $=1$, KCEITS

WRITE (2. $16903 \mathrm{E}(1), F(1), X A D D P(1), X$ XADDC $(1), \operatorname{XSPECP}(1), \operatorname{XSPECC}(1)$

530 ConTINUE

if ( J OUART. Lo. is NAITE 12,1230

IF ( IQUART, EQ. 1$)$ HRITE $(2,1240)$

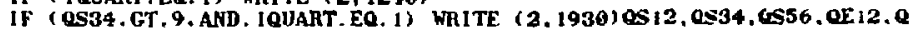
1534.0506

IF (OS34.LT. 10. AND. IQUART. EG, 1) WRITE (2, 1940) OS12, 0S34.0S56,0 12 . 10534,0556

IF ( IOUART, EQ. 1) WRITE (2. 1950) FYEAR

IF ( IOUART, EQ. I)

DO $54 B$ I $=1$. NCHITS

840 CONTINUE

IF ( IQUART. EQ. 1) WRITE $(2,1970)$

IF ( 1QUART, EQ, 1$)$ WRITE $(2,1989)$

DO $550 \quad I=1$, NCBITS

IF ( IOUAAT. EQ. 1) WRITE (2,1990) E( I), F( I), OXADDC I , OXSPECC ( )

B50 CONT INUE

WRITE $(2.1410$

WhiTE $(2,1350$

WRITE ( 2,1360$)$ CLASS 10, ClaSS IB

WR ITE $(2,1370)$ CLASS2U, CLASS2B

WRITE (2, 1380) CLASSAU, CLASS3B

WRITE $(2,1390)$ CLASS 0 , CLASS $4 B$

WRITE (2, 1496$)$ CLASSEU, CLASS5B

D0 $5601=1,27$

$K=2+1$

REWIND

560 cortinus

570 READ $(2 \theta, 1660)($ DATA $(1), I=1,13)$

IF $(\mathrm{EOF}, 20) 590,58 \theta$

580 WRITE (2, IB60) ( DATAC 1 , $1=1,13)$

co To 376

590 COKTIFU:

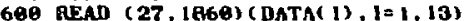

IF (E.F. 27) 626.610

610 WAITE $(2,1868)($ DATA $I), I=1,13)$

G0 T0 600

620 CokTIKuE

HAITE (2.1768)

IF ( IADD. IE. 1.ANT.LIST. HE, 1$)$ GO TO 650 


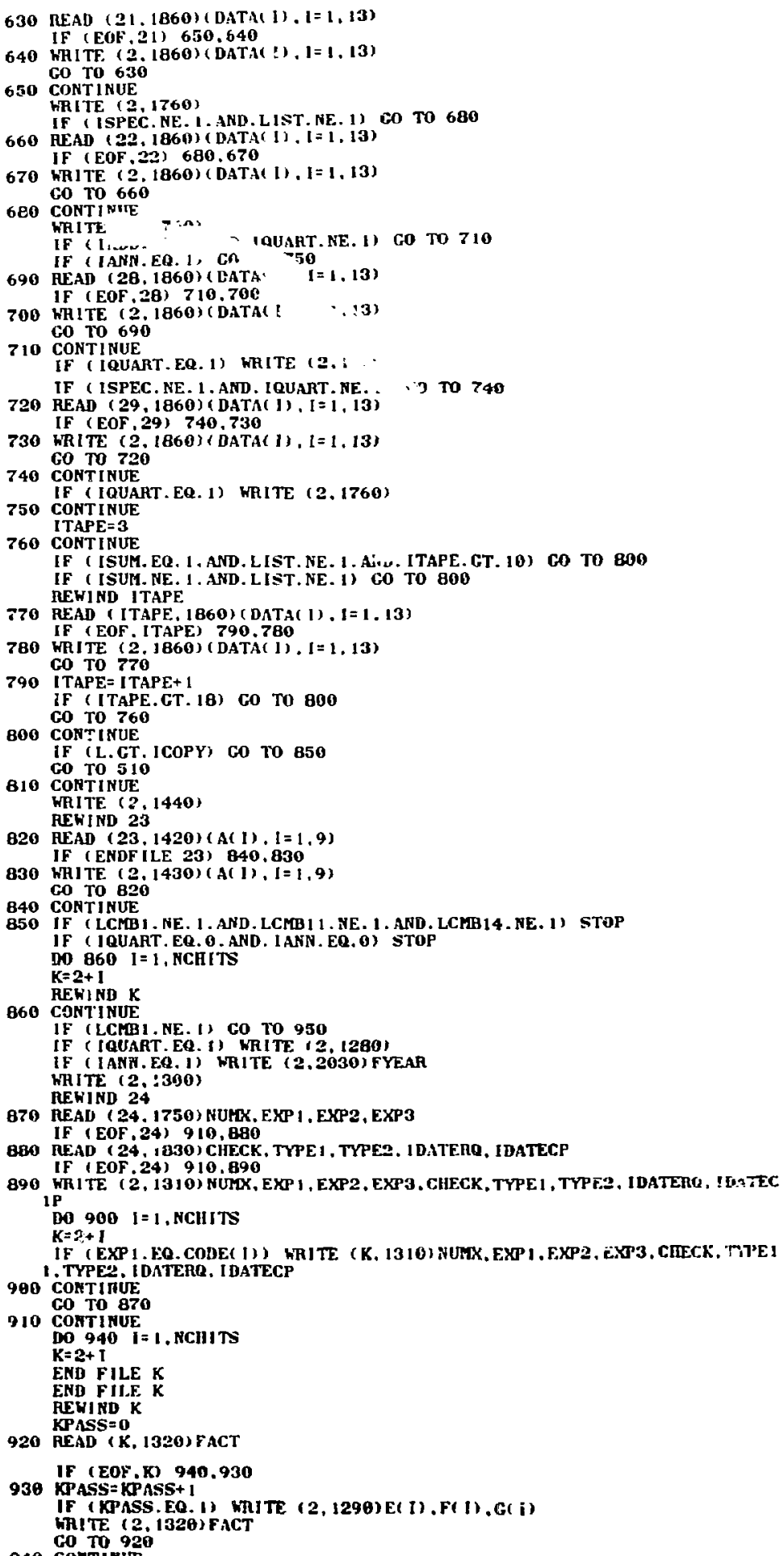


950 If (LCMH! I.NF. I ) FO TO 1980

IF ( IQUNAT EQ. I) MILTE $(2,1390)$

if (INNA. EQ. I) HAITE $(2,2040)$ FYENR

HR1TE 12,130

$10960 \quad i=1$, NCl. ITS

$K=2+1$

960 FEWIND $K$

REWIND 25

970 READ $(25,1750)$ NURX, EXP 1, EXP2, EXP3

IF (EOF 25$) 1010,780$

980 READ (25. 1830 ) CHECK, TYPE, , TYPE, . IDATERQ, IDATECP

IF (EOF, 25) 1010,990

990 HTITE (2,1310) HUPX, EXP1 EXP2, EXP3 CHECK, TYHE1, TYPEO, IDATERA. IDATEC $1 \mathbf{P}$

MO $10001=1$, NCNITS

$\mathrm{K}=2+1$

IF ( EXP I EQ. CODE $(1)$, WAITE $(K, 1310)$ NUTX, EXP I, EXP2, EXP3, CHFCK, TYPE:

1. TYPEO, JDATERQ, JDATECP

1000 CONTINUE

CO TO 970

1010 CONTI NUT

DO $1040 \quad I=1$, NCHITS

$K=2+I$

EHD FII.E $K$

END F ILE $K$

REWIND $K$

KPASS $=0$

1020 READ $(K, 1320) F A C T$

IF (EOF, K) 1040.1030

1030 KPASS= KPASS+1

IF (KPASS EQ. 1) WRITE $(2,1880)$ E( 1$), F(J), G(I)$

WIITE ( 2 . 1320) F ACT

co To 1020

1949 CONTINUE

1050 CONTINUF.

IF (LCMBI4. NF. I) Go TO 1130

if (LCABI4. MF... I) 6 ) To 1130

IF (IANN. FQ. I) HRITE (2.2050) FTEAR

IF ( IANN. EQ. I)

OO $1060 i=1$. HF. IT

$\mathrm{K}=\mathbf{2}+\mathbf{1}$

1060 AEWIND $K$

RENIND 26

1670 AEAD $(26,1750)$ NUHX, EXP 1, EXP2, EXPa

IF (EOF.26) 1110,1080

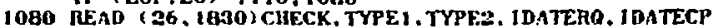

IF (EOF.26) 11001090

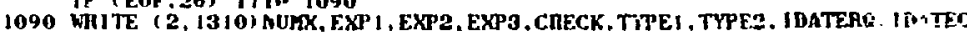
IP

DO $11001=1$, NCHITS

$K=2+1$

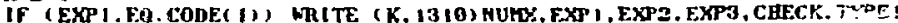
1, TYPE? IDATERO. IDATECP

100 cort Ifirs

Go to 1070

1110 Continuf.

Bo $11401=1$, NCIITS

$\mathrm{X}=\mathbf{2 +}$

END FILE $K$

END FILE K

NEWIND $K$

1120 READ ( K. 1320) FACT

If $(E D F, K)(140, t 13 a$

1100 KPASS KPASE+

If (KPASS. E. 1) WITE (2, 1890) E(I), F( I), G( I)

WHITE $\{2,1320\}$ FACT

GO TO II:34

1140 Corrt Inte

1150 s70p

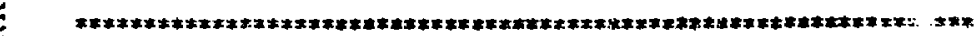

*Fonhats:

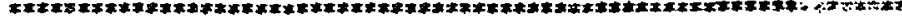

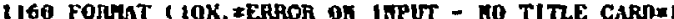

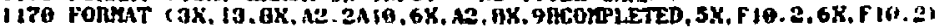

100 FoRHat $(1)$ )

190 Fon 1312

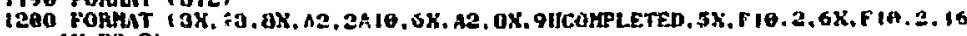
IX. Fa.2)

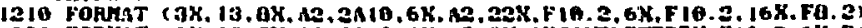

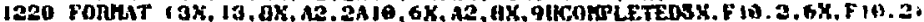

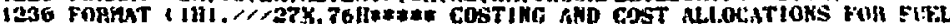

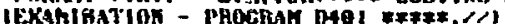

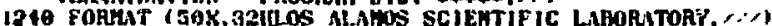

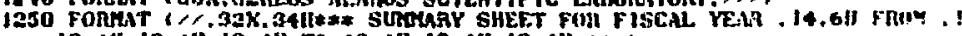
$12,111-12,111-12,411$ m $12,111-12,18-12,411$ **1,

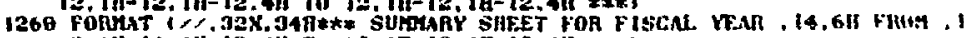

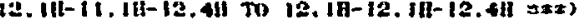


1270 PORMAT ( 1 H $1, / /, 3 \mathrm{X}, 7 \mathrm{~A} 10,27 \mathrm{X}, 5 \mathrm{HUATE}, 12,1 \mathrm{~B}-12,1 \mathrm{II}-12)$

1289 FOHMA ( 1 H1,29X,74H*** LISTIHC OF EXAMINATIONS COMPLETED BY CHB- 1 IDUR INC QUARTERLY PERIOD $* * *,(X)$

1299 FORMAT ( IRI, 5X, 23BCMB-1 EXAMINATIONS FOR, 3A10, //,9B LASI. NO, ,5X, 127 BEXPEA I IENTEA IDENTIF ICATION, $5 X, 23$, 2EXAMINATION IFSCAIPTION, $4 X, 9 \mathrm{H}$ EREQUESTED, $4 \mathrm{X}, 9 \mathrm{BCOMPLETED},>$

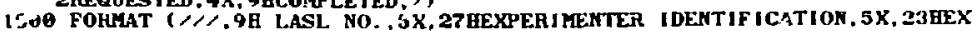
1 AFI KAT ION DESCR IPTIOH, 4X, 9EREQUESTED, 4X,9HCOHPLETER, $\rightarrow$

1310 FORHA (6H )

1320 FORMAT (10A16)

1330 FORMAT (IH $1,27 X, 75 H * * *$ LISTING OF EXIMINATIONS COMPLTED BY CHB- 1 1 DURING QUARTERLY PERIOD $\neq * \pm, / /$ )

1340 FORHAT ( 1 H $1.27 \times, 75 H * * *$ LISTING OF EXAYIMAT1ONS COKPLETED BY CMB- 14 1 DURING OUARTERIY PERIOD $* * * \ldots, \ldots)$

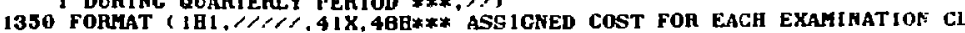
1ASS ***, $/ 1,1)$

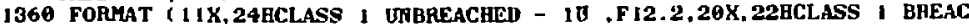
IHED - IB,Fi2.2,

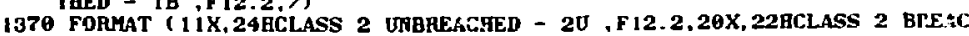
I HED - $2 B, F 12.2, \prime$

1380 FOAMAT ( 1 ix,24BCLASS 3 UHBPEACHED - 3U,F12.2.20X,22HCLASS 3 BHE.AC 1EED - 3B, Fi2.2.,

1390 FORHAT ( 1 1 X.24BCLASS 4 UNBREACHED - 4U, F12.2,20X.22日CLASS 4 BIIEAC 1 HED - 4B, F $12,2, C$

1400 FORMAT ( $11 \%, 24$ HCLASS 5 UNBREACRED - 5U, F $12.2,29 X, 22 R C L A S S$ S BFEAC $1 \mathrm{KED}-\mathrm{SB}, \mathrm{F} 12.2,3$

1410 TORHAT $(, 1, /$,

1420 PORHaT (9A10)

1430 FORMAT $(1 \mathrm{X}, 9 \mathrm{~A} 1 \mathrm{\theta})$

1446 FORMAT (1Hi, //, 16x, 32RLISTING OF DATA OF FUEL PII LIST, //)

1450 FORMAT (BA10)

1460 FORHAT (10I 1)

1470 FORMAT (F 10.3$)$

1480 FORMAT ( $3 X,[3,15 X, A 2,2 A 10,5 X, A 4,2 A 10,8 X, F 12.2,12 X, F 12.2)$

1490 FORMAT ( 1 HI,, , 33X,64H*** SUMGARY SHEET FOR SPECIA: EXAMINATIONS 1COMPLETED TO DATE ***, $/,, 1 X, 8$ KLASL NO . 8X, 19HP1A IDENTIFIEATION. $215 X, 11$ IIEXAMINATION, $19 X, 14$ HPROJECTED COST, 12X, 1 1HACTUAL COST, $>$,

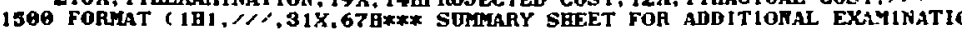
INS COMPLETED TO DATE $* * *, ノ / ., 1 \times, 8 H L A S L$ NO. BX, 19H PIA IDENTIFICATI 20N, 15X, I IHEXAHIRATIOK, 19X, 14GPROJECTED COST , 12X, 11HACTUAL COST,

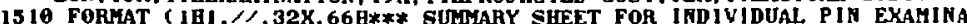
ITION COSTS TO DATE $* * *, \angle$,

1520 FORHAT (/70X, 2 HPRESERT, tOX, 5HTOTAL)

1530 FORLAT ( $1 \mathrm{X}, 8 \mathrm{HLASL}$ NO, 6X, 18HP IN IDENTLFICATION, 8X, 7H CLASS , 8X,6HS 1TATUS, BX, BHFY TOTAL, BX, BHALL FYS , 10X, 1BHCLASS COST OVEARUN,

1540 FORHAT (3A10,A2, BX, F 10,2)

1550 FORHAT (BA 10)

1560 FORHAT (///, 3X,6B LASL $5 \times, 13$ HEXPEAIMETTTA, IOX, 11 HEXAHIFATION, $16 \mathrm{X}$ 1,9 HESTIMATED, $5 \times, 2 \mathrm{HO} / 0$ OF $, 5 \mathrm{X}, 7 \mathrm{H}$ TOTAL .3X,6HO 0 OF)

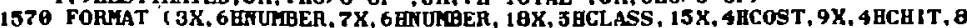
I $X, 4 \mathrm{HCOST}, 8 \mathrm{X}, 4 \mathrm{HCBIT}, / /$ )

1580 FORYAT $(, .66 \mathrm{X}, 12 \mathrm{H}$ TOTAL, $S)=, 4 \mathrm{X}, \mathrm{F} 12.2 .3 \mathrm{~K}, \mathrm{~F} 12.2)$

1590 FORYAT $(/, 76 \mathrm{X}, 14 \mathrm{H}$ DIFFEREHCE $=, \mathrm{F} 12.2)$

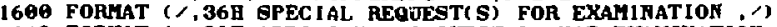

1610 FORFAT ( $/, 39$ H ADDITIONAL REOUEST(S) FOR EXAYINATIOH,$)$

1620 FORFAT $(/ / /, 5 X, 16$ HTUMBER OF CHIT $=, 12, / /)$

1630 FORHAT $(<, I X, 2 A 16,5 X, F 10,2,8 X, F 10.2, i 0 X, F 7.2,16 X, F 12.2,9 K, F 7.2 .9 X$ 116)

1640 FORHAT $(/ /, 55 \times$, IIB*** GROUP BUFHARY ***,//)

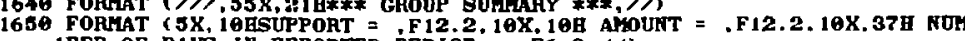
IBER OF DAYS IN REPORTED PERIOD $=F 6,2,1 / 3$

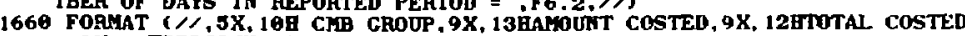
$1,9 \mathrm{X}, 16$ EPERCETT OF TOTAL, $/ O$

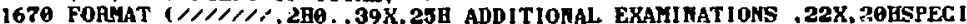
IAL EXAMI NAT TONS)

1689 FORHAT (5X, I3HORGATITATIOA, 22X, 9HPROJECTED, 13X, 6HCOQTED. 18X, 9HPRO 1 IECTED, 10X, 6BCOSTED. $/ 1$

1690 FORHAT (1X,2A10,16X,F 12.2, BX,F12.2, 14X,F12.2, 5X,F12.2.,

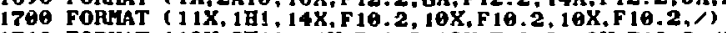

1710 FORMAT (10X, 2B1 $14 \mathrm{X}, \mathrm{F} 10.2,10 \mathrm{X}, \mathrm{F} 10.2,10 \mathrm{X}, \mathrm{F} 10.2, /)$

1720 FORHAT (10X, 2E14, I4X,F10.2, 10X,F10.2, 10X,F10.2, /)

1730 FORLAT $(1 \mathrm{H} 1, /, 20 \mathrm{X}, \mathrm{BA} 1 \theta, / / /)$

1740 FORHAT ( $3 X, 13, B X, A 2,2 A 16,6 X, A 2,22 X, F 10,2,6 K, F 10.2)$

1756 FORMAT (13,3X,A2, $2 A 10,8 \times, 92, F 10.3)$

1766 HORMAT ( $/, 10 \times, 97$ BTHE DOL 1 IIOUS DUE TO CHANGES IN THE BUPPORT ING ACTIVITIES,

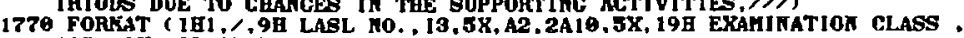
1A2, 10X, 198 PAEVIOUS FY TOTAL . F12.2, $/, 1$

1780 FORMAT (6X, 13H EXAMINATION, 1 iX, 16B DATE REOUESTEN, 4X, I4EDATE COFI ILETED , 6X, IOHWORK UI ITS, 6X, 12H EST COST \&X, IOHTOTAL COST, / I

1790 FORHAT (A4,2A16,6X, I6, 6X, 16,6X, 16,6X, F6.2,6X, 12)

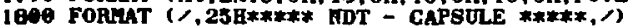

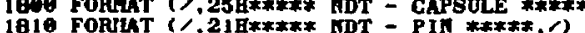

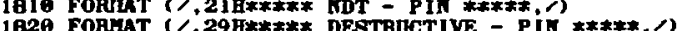

1830 FORIAT (IX,A4,2A16,9X, I6, I2X, I6, I2X, F8,2,4X,F12, 2, aX, F 12, 2)

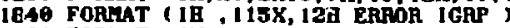

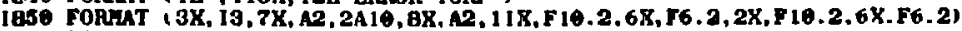

186. PORMAT (13A18) 
1670 FORMAT (//, 5X, 12HORGAIIZATION, IOX,3HFY, I4,5H CHIT, 5X, 9ECORYITTEO

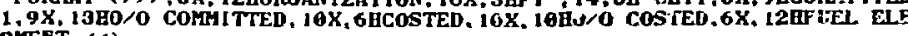
ZHENT,

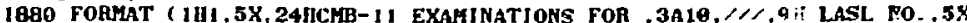
I, 27 HEXPEHIMENTER IDENTIF ICATION.5X. 23HEXAMINATION DESLAIPTIOH, 4 X, 9 2HEOUESTED $4 X$, 9 HCOHPLETED,

1896 FORHAT (1Hi,5X, 24ICHB- IS EXAMINATIONS FOR 3A1G, /, 9 H LASL RO..5X 1,27 HEXPERIMENTER IDENTIFICATION, 5X.23HEXAMINATION DESCAIPTIUH.4X.9 2HAFOUESTED, 4X, 9HCOKPLETED.

1990 FORHAT $1 \mathrm{Hi}, / / 24 \times .83 \mathrm{H} * * *$ SUMLAY SHEET FOR ADDITIORAL EXAMIHATJO INS CORPLETED DURING QUARTERLY PERIOD $* * * . / /, 13 \times, 8 H L A S L$. NO. . BX. 19H \& PIN IDFNTIF ICATION, ISX, 1 I BEXAHINATION, $19 \mathrm{X}, 1$ I HACTIAL COST,

1910 FOAMAT $(1 \mathrm{HI}, / /, 25 \mathrm{X}, 80 \mathrm{H} * * *$ SUMMARY SHEET FOR SPEC I AL EXAYINATIOFS 1 COMPLETED DURING QUARTERIY PERIOD ***, $/ /, 13 \%$, AHLASL NO. 8X, 19P PI 2N IDENTIF ICATION, ISX, I IHEXAMINATION, I9X, I I HACTUAL GOST, $;$

1920 FGRHAT ( 1 H 1 , $27 \times$, 5 HE* SUE ITED DURIFG QUABTERLY PERIOD $* * *, /, j$

1930 FORMAT ( $/ 29 \times, 39 H * * *$ SULPARY SHEET FOR QUARTERLY PERIOD , $6 \mathrm{H}$ FROH $1,12,1 \mathrm{H}-12,1 \mathrm{H}-12,4 \mathrm{H}$ TO $12,1 \mathrm{H}-12,1 \mathrm{H}-12,4 \mathrm{H} * * *)$

1940 FOTHAT ( $r .29 \times, 39 H * * *$ SUMHARY SHEET FOR QCARTERLY PERIOD , $6 H$ FROH $1,12,1 \mathrm{H}-11,1 \mathrm{H}-12,4 \mathrm{H}$ TO $12,1 \mathrm{H}-12,1 \mathrm{H}-[2,4 \mathrm{H} * * *)$

1950 FOAMAT ( //, 20X, 12HOAGAKIZATIOH 14X, 3HFY, 14, 5H CHIT, 12X, 6HCOSTED $112 \times, 10 \mathrm{O}, 0$ COSTED,,$J$

1960 FIRHAT $(,-15 X, 2 A 1 \theta, 11 X, F 10,2,11 X, F 10,2,12 X, F Z .2)$

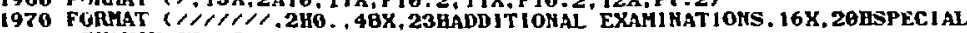
1 EXAMINATIONS)

1980 FOAMAT ( 20X, I2HORGAN IZAT ION, 26X, 6BCOSTED, 3 I X, 6HCOSTED, $/ /)$

1990 FOAMAT ( 15X. 2A10, 19X, F 10.2,29X, F10.2 $)$

2900 FOAMAT ( 16X, 13, 15X, A2, 2A10,5X, A4, 2A10, gX, F 12.2)

$20: 0$ FORHAT (69X, 36H NUMBER OF DAYS IN QUARTER PERIOD = F6.2, $/ /$ )

2020 FORMAT (1H1 32X G7B** SIMARY SHEET FOA INDIVIDUAL PINS COTPLF ITED DURING FISCAL YEAR , 14,4B ***, /,)

2030 FORHAT (1H1, 28X,66H*** LIST1NG OF EXAMINATIONS COHPLETEO BY CHBIDURING FISCAL YEAR, $14,4 \mathrm{4} * * *, \pi)$

2040 FORHAT (1HI.27X.67H*** LISTING OF EXAHINATIONS COMPLETED BY CMB- I t DURING FISCAL YEAR, $14,4 \mathrm{E} * * *, 1 / 1$

2050 FORMAT (IHI.27X,67H*** LISTING OF EXAHINATIONS COMPLETED BY CHB- 14 1 DURIRG FISCAL YEAR 14, $9 \mathrm{H} * * *, / 2)$

2060 FORHA I

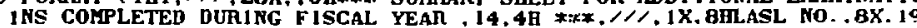
2A PIN IDENT IF ICATION, 15X, 1 I HEXAMI IATION, 19X, 14HPROJECTED COST. 12X. 311 HACTUAL COST,, )

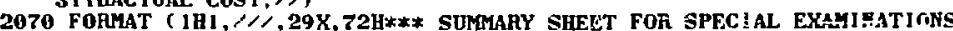
1COHPLETED DUAING FISCAL YEAR I4, 4H ***, $/, 1 \mathrm{X}$, \&HLASL NO., BX. 19H 2IN IDENT1F ICATIOK, 15X, 11HEXAMINATION, 19X, 14HPROJECTED COST, 12X $11 \mathrm{H}$ 3ACTUAL COST, /ノ) 
APPENDIX $\mathbf{G}$

**** COSTING AND COST ALLOCATIONS FOR FUEL EXAMINATION ${ }^{2}$ - PROGRAM D401****

LOS ALAMOS SCIENTIFIC LABORATORY

*** SUMMARY SHEET FOR FISCAL YEAR 1975 FROM 7-1-74 TO 6-30-75***

$\begin{array}{rccccrr}\text { ORGANIZATION } & \text { FY } 1975 \text { CHIT } & \text { COMMTTTED } & \text { O/O COMMITTED } & \text { COSTED } & \text { O/O COSTED } & \text { NO. PINS } \\ \text { EXPERIMENTER A } & 238000.00 & 275173.66 & 115.62 & 230582.81 & 96.88 & 115 \\ \text { EXPERIMENTER B } & 102000.00 & 108554.41 & 106.43 & 77756.68 & 76.23 & 8 \\ \text { EXPERIMENTER C } & 30000.00 & 579584.93 & 193.19 & 374600.60 & 124.87 & 107 \\ \text { EXPERIMENTER D } & 360000.00 & 976309.64 & 197.71 & 317059.91 & 88.07 & 90\end{array}$

ORGANIZATION
EXPERIMENTER A
EXPERIMENTER B
EXPERIMENTER C
EXPERMENTER D

\section{ADDITIONAL EXAMTNATIONS PROJECTED COSTED}

41536. $18 \quad 41536.18$

24122. $70 \quad 25122.70$

50032. 72 50032.72

$4008950 \quad 40039.50$

\begin{tabular}{rr}
\multicolumn{2}{c}{ SPECLAL EXAMTNATIONS } \\
PROJECTED & COSTED \\
40667.47 & 37871.80 \\
5296.48 & 2500.81 \\
22105.67 & 17404.30 \\
25180.39 & 23099.95
\end{tabular}

${ }^{a}$ Cost figures are hypothetical with the total of the experimenter's chit arbitrarily set to $\$ 1$ million.

APPENDD H

**** COST 2 NG AND COST ALLOCATIONS FOR FUEL EXAMINATION ${ }^{2}$ - PROGRAM D401****

LOS ALAMOS SCIENTIFIC LABORATORY

*** SUMMARY SHEET FOR QUARTERLY PERIOD FROM 4-1-75 TO 6-30-75 ***

$\begin{array}{lccc}\text { ORGANIZATION } & \text { FY } 1975 \text { CHIT } & \text { COSTED } & \text { O/O COSTED } \\ \text { EXPERIMENTER A } & 238000.00 & 54689.68 & 22.98 \\ \text { EXPERIMENTER B } & 102000.00 & 35530.64 & 34.83 \\ \text { EXPERIMENTER C } & 300000.00 & 90077.35 & 30.03 \\ \text { EXPERIMENTER D } & 360000.00 & 123266.90 & 34.24\end{array}$

ORGANIZATTON
EXPERIMENTER A
EXPERIMENTER B
EXPERIMENTER C
EXPERIMENTER D

ADDITIONAL EXAMTNATIONS COSTED

22221. 80

24122. 70

6725. 10

24530. 79
SPECIAL EXAMINATIONS COSTED

13036. 34

0.00

159. 35

11177. 2\&

\footnotetext{
a Cost figures are hypothetical with the total of the experimenter's chit arbitrarily set to \$I million.
} 


\section{APPENDIX I}

*** SUMMARY SHEET FOR INDIVIDUAL PIN EXAMNATION $\operatorname{cosTs}^{\mathrm{a}}$ TO DATE***

\begin{tabular}{|c|c|c|c|c|c|c|}
\hline LASL NO. & $\begin{array}{c}\text { PIN } \\
\text { DENTIFICATION }\end{array}$ & CLASS & STATUS & $\begin{array}{l}\text { PRESENT } \\
\text { FY TOTAL }\end{array}$ & $\begin{array}{l}\text { TOTAL } \\
\text { ALL FYS }\end{array}$ & $\begin{array}{l}\text { CLAES COST } \\
\text { OVERRUN }\end{array}$ \\
\hline 102 & ED-7\$1 & & COMPLETED & 773.42 & 773.42 & \\
\hline 107 & ED-591 & $1 \mathrm{U}$ & COMPLETED & 0.00 & 3598.49 & 498.49 \\
\hline 110 & ED-002 & & COMPLETED & 0.00 & 4356.80 & \\
\hline 111 & ED-602 & & COMPLETED & 773.42 & 773.42 & \\
\hline 115 & ED-2N-34 & 1B & COMPLETED & s1. 56 & 3273.03 & \\
\hline 117 & EC-Q21 & & COMPLETED & 110.71 & 110.71 & \\
\hline 118 & EC-P21 & & COMPLETED & 110.71 & 110.71 & \\
\hline 132 & ED-73-B & & COMPLETED & 0.00 & 0.00 & \\
\hline 135 & ED-1N-34 & $\mathbf{1 B}$ & COMPLETED & 0.00 & 1331.81 & \\
\hline 140 & ED-54 & $1 \mathrm{~B}$ & COMPLETED & 0.00 & 8063.46 & 3463.46 \\
\hline 141 & $2 D-46$ & 1B & COMPLETED & 31.56 & 13431.49 & 8831.49 \\
\hline 142 & ED-94 & $\mathbf{1 B}$ & COMPLETED & 0.00 & 6560.85 & 1960.85 \\
\hline 143 & ED-50 & & COMPIETED & 0.00 & 0.00 & \\
\hline 144 & ED-51 & $4 \mathrm{~B}$ & & 8067.82 & 8067.82 & \\
\hline 145 & ED-2-3 & $1 \mathrm{~B}$ & COMPLETED & 31.56 & 8888.83 & 4288.83 \\
\hline 146 & $E D-2-2$ & $5 B$ & COMPLETED & 11598.59 & 14025.25 & \\
\hline 148 & ED-5-3 & $\mathbf{5 B}$ & COMPLETED & 31.56 & 4715.69 & \\
\hline 149 & ED-B63 & $5 B$ & COMPLETED & 0.00 & 13638.23 & \\
\hline 150 & ED-88 & $5 \mathbf{U}$ & & 0.00 & 1601.31 & \\
\hline 151 & ED-39 & & COMPLETED & 773.42 & 773.42 & \\
\hline 152 & ED-49 & $5 U$ & COMPLETED & 811.38 & 7001.25 & \\
\hline 153 & ED-79 & $3 \mathrm{U}$ & COMPLETED & 3545.93 & 10037.99 & \\
\hline 154 & ED-89 & $\mathbf{5 B}$ & & 8396.15 & 10652.13 & \\
\hline 156 & ED-601 & $5 B$ & & 7394.10 & 9650.08 & \\
\hline 157 & ED-011 & $3 \mathbf{U}$ & & 0.00 & 3287.52 & \\
\hline 158 & ED-311 & $3 U$ & & 0.00 & 3287.52 & \\
\hline 159 & ED-411 & 3B & & 0.00 & 2255.98 & \\
\hline 160 & ED-581 & $1 U$ & & 0.00 & 1601.31 & \\
\hline 161 & ED-681 & $1 U$ & & 0.00 & 1601.31 & \\
\hline 162 & ED-202 & $1 \mathrm{U}$ & & 0.00 & 1601.31 & \\
\hline 163 & ED-203 & $1 \mathrm{U}$ & COMPLETED & 0.00 & 1601.31 & \\
\hline 164 & ED-204 & $1 U$ & & 0.00 & 1601.31 & \\
\hline 165 & ED-205 & 10 & & 0.00 & 1601.31 & \\
\hline 166 & EC-I8F & $3 U$ & COMPLETED & 0.00 & 9005.74 & \\
\hline 167 & EC-I9J & $3 U$ & COMPLETED & 0.00 & 8916.99 & \\
\hline 168 & EC-I10 & $3 \mathbf{U}$ & COMPLETED & 0.00 & 8049.20 & \\
\hline
\end{tabular}

${ }^{a}$ Cost figures are hypothetical; they were chosen only as examples of the type of output from the computer program. 


\section{APPENDLX $J$}

*** SUMMARY SHEET FOR INDIVDUAL PINS COMPLETED DURING QUARTERLY PERIOD ***

\begin{tabular}{|c|c|c|c|c|c|c|}
\hline LASL NO. & $\begin{array}{c}\text { PIN } \\
\text { DENTIFICATION }\end{array}$ & CLASS & STATUS & $\begin{array}{l}\text { PRESENT } \\
\text { FY TOTAL }\end{array}$ & $\begin{array}{l}\text { TOTAL }^{\mathbf{a}} \\
\text { ALL FYS }\end{array}$ & $\begin{array}{c}\text { CLASS COST }^{a} \\
\text { OVERRUN }\end{array}$ \\
\hline 144 & ED-51 & $4 \mathrm{~B}$ & COMPLETED & 11796.15 & 11796.15 & \\
\hline 150 & ED-88 & $5 \mathrm{U}$ & COMPLETED & 0.00 & 1601.31 & \\
\hline 160 & $\mathrm{ED}-581$ & $1 U$ & COMPLETED & 0.00 & 1601.31 & \\
\hline 161 & ED-681 & $1 U$ & COMPLETED & 0.00 & 1601.31 & \\
\hline 162 & $\mathrm{ED}-202$ & $1 U$ & COMPLETED & 0.00 & 1601.31 & \\
\hline 164 & $E D-204$ & $1 \mathrm{U}$ & COMPLETED & 0.00 & 1601.31 & \\
\hline 165 & $\mathrm{ED}-205$ & $1 \mathrm{U}$ & COMPLETED & 0.00 & 1601.31 & \\
\hline 183 & $E B-100$ & $5 B$ & COMPLETED & 46256.51 & 53455.66 & 21455.66 \\
\hline 261 & ED-562 & $5 B$ & COMPIETED & 29438.27 & 31134.51 & \\
\hline 262 & $\mathrm{ED}-622$ & $\mathbf{5 u}$ & COMPLETED & 13066.86 & 13066.86 & \\
\hline 287 & EB-41 & $5 U$ & COMPLETED & 21044.64 & 25645.90 & \\
\hline 289 & EB-61 & $5 U$ & COMPLETED & 23792.05 & 28393.31 & \\
\hline 311 & EB-60 & $5 U$ & COMPLETED & 24641.10 & 26337.34 & \\
\hline 324 & ED-3-2 & $4 U$ & COMPLETED & 9194.35 & 12028.71 & \\
\hline 329 & ED-FW6 & $4 B$ & COMPLETED & 9854.29 & 11188.58 & \\
\hline 335 & ED-842 & $5 B$ & COMPLETED & 33200.00 & 35247.01 & 3247.01 \\
\hline 433 & ED-331 & $1 U$ & COMPLETED & 4988.00 & 4988.00 & 1888.00 \\
\hline 434 & $\mathrm{ED}-431$ & $1 U$ & COMPLETED & 4988.00 & 4988.00 & 1888.00 \\
\hline
\end{tabular}

THE DOLLAR VALUES MAY VARY BETWEEN ACCOUNTENG PERIODS DUE TO CHANGES IN THE SUPPORTING ACTIVITIES

${ }^{a}$ Cost figures are hypothetical; they were chosen only as examples of the type of output from the computer program. 
APPENDTX K

\begin{tabular}{|c|c|c|c|}
\hline LASL NO. & PIN IDENTIFICATION & EXAMINA TION & ACTUAL $\operatorname{COST} T^{a}$ \\
\hline 183 & $E B-100$ & SEQ. SURFACE PREP. & 1387.74 \\
\hline 272 & EC-24 & NÁ PISTILLATION & 132.79 \\
\hline 327 & ED-102 & NA DISTILLATION & 132.79 \\
\hline 329 & ED-666 & NA DISTILLATION & 132.79 \\
\hline 334 & ED-902 & NA DISTILLATION & 132.79 \\
\hline 335 & ED-842 & SEQ. SURFACE PREP. & 1387.74 \\
\hline 408 & EA-A81 & TWO DIM GAMMA SCÃN & 931.89 \\
\hline $40 y$ & $\mathrm{EA}-\mathrm{A}-42$ & TWO DIM GAMMA SCAN & 931.89 \\
\hline 410 & $E A-A-63$ & TWO DIM GAMMA SCAN & 931.89 \\
\hline 450 & $E C-43$ & SCRIBE REF. MARKS & 13.28 \\
\hline 451 & $\mathrm{EC}-44$ & SCRIBE REF. MARKS & 13.28 \\
\hline
\end{tabular}

THE DOLLAR VALUES MAY VARY BETWEEN ACCOUNTING PERIODS DUE TO CHANGES IN THE SUPPORTING ACTIVTTIES

${ }^{\text {a }}$ Cost figures are hypothetical; they were chosen only as examples of the type of output from the computer program. 
A PPENDIX L

LASL NO. 419 EC-789-C EXAMINATION CLASS U

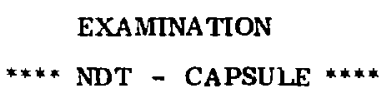

PROFILOMETRY, OPT.

121774

62475

121774

$-0$

GROSS GAMMA SCAN

SPECT. GAMMLA SCAN

EDDY CURRENT SCAN

**** DESTRUCTIVE - PIN ****

WIRE WRAP REMOVAL

FISSTON GAS SAMPLNG

FISSION GAS ANALYSIS

SECTIONING

121774

121774

61975

61975

121774

62075

121774

121774

121774

121774

60975
-0
-0
-0

30.

170.

3.

TOTAL (S)

DIFFERENCE $=2566.13$
132. 2 A $132.2 \times$

66. $40 \quad 66.40$

22.1322 .13

22.1322 .13

22.1322 .13

$387.72 \quad 287.72$

13. $29 \quad 13.29$

$150.50 \quad 150.50$

69.8969 .59

$150.50 \quad 150.50$

$150.50 \quad 150.50$

$30.98 \quad 30.99$

46. 5900

$177.06 \quad 0.00$

177.06. 0.00

$\begin{array}{rrr}75 . & 66.40 & 66.40 \\ 25 . & 22.13 & 22.13 \\ 325 . & 287.72 & 287.72 \\ 610 . & 540.03 & 0.00 \\ 16 . & 372.76 & 372.76 \\ 30 . & 698.92 & 698.92 \\ 50 . & 44.26 & 44.26\end{array}$

\footnotetext{
${ }^{a}$ Work units are related to actual dollars by a conversion factor unique to each examining group.

b

Cost figures are hypothetical; they were chosen only as examples of the type of output from the computer program.
} 